\title{
On directed compact percolation near a damp wall
}

 \\ ${ }^{1}$ Department of Mathematics and Statistics, The University of Melbourne, Victoria 3010, \\ Australia \\ 2 Department of Mathematics, Royal Holloway, University of London, Egham, Surrey \\ TW20 0EX, UK \\ ${ }^{3}$ Department of Mathematics, University of British Columbia, BC V6T-1Z2, Canada \\ E-mail: h.lonsdale@ms.unimelb.edu.au,r.brak@ms.unimelb.edu.au,j.essam@rhul.ac.uk, \\ a.owczarek@ms.unimelb.edu.au and andrewr@math.ubc.ca
}

Received 18 December 2008, in final form 5 February 2009

Published 24 February 2009

Online at stacks.iop.org/JPhysA/42/125001

\begin{abstract}
A percolation probability for directed, compact percolation near a damp wall, which interpolates between the previously examined cases, is derived exactly. We find that the critical exponent $\beta=2$ in common with the dry wall, rather than the value previously found in the wet wall and bulk cases. The solution is found via a mapping to a particular model of directed walks. We evaluate the exact generating function for this walk model which is also related to the ASEP model of traffic flow. We compare the underlying mathematical structure of the various cases previously considered and this one by reviewing the common framework of solution via the mapping to different directed walk models.
\end{abstract}

PACS numbers: $05.50 .+q$, 05.70.fh, 64.60.ah

(Some figures in this article are in colour only in the electronic version)

\section{Introduction}

Percolation is a key problem in statistical mechanics, and there are many practical applications for percolation theory-including the modelling of the spread of epidemics [7] and forest fires [19], of metal-insulator transition [1] and of the flow of sand [17]. Percolation displays a fundamental critical phenomenon and deep recent progress [9] has been made to mathematically expound its behaviour in two dimensions. The basic model has been modified in many ways both to model different systems and in the search for integrable systems. While for self-avoiding walks the introduction of directness leads to various solvable models its imposition alone on percolation does not lead to any such solvable cases. However, by adding the further condition of 'compactness' a solvable lattice model ensues [8]. Domany and Kinzel [8] introduced directed, compact percolation and found exact expressions for the percolation probability, the cluster length distribution and the associated critical exponents in the bulk far 
from any confining walls. Essam [10] extended this work to biased growth and to mean-cluster size and length, finding exact recurrence relations for length and size distributions, the closed form for the relation for the moment generating function of the length distribution, and the first three moments for the size distribution. Following on from this, non-nodal clusters and the cluster size distribution were investigated by Essam and TanlaKishani [13].

The effect of adjacent interacting walls is a key topic in critical phenomena and the effect of the presence of a wall in directed, compact percolation was first considered by Essam and TanlaKishani [14]. They considered a so-called wet wall where the sites of the wall are all deemed to be occupied. They found expressions for the percolation probability, the mean length of finite clusters and the mean-cluster size, and so discovered that the critical behaviour of the percolation probability in the wet case is the same as in the bulk case [8]. The opposite case of a dry wall, where the sites along the wall are fixed to be unoccupied, was considered by several authors. Here the critical behaviour departs from the bulk model.

An exact form of the percolation probability in the dry-wall case was conjectured by Bidaux and Privman [2] and derived by Lin [18], who found that the critical exponent for the percolation probability, $\beta$, changed from the bulk value $\beta=1$ to $\beta=2$ in the dry case. Essam and TanlaKishani [14] generalized this result to include the possibility of a bias towards the wall or a bias away from the wall. Essam and Guttmann [11] found an exact expression for the mean-cluster size below the critical point and used differential approximants to conjecture that the average cluster size above $p_{c}$ and the mean-cluster length satisfy second-order differential equations. Brak and Essam [5] used a mapping to a directed walk problem to derive the percolation probability and used the Zeilberger algorithm [20,21] to find the mean length and number of contacts (occupied sites adjacent to the wall) in the dry case- these results involved elliptic integrals.

Given that the critical behaviour varies from wet to dry, and also that the dry case demonstrates quite different mathematical behaviour, it is of interest to examine a model which interpolates between dry and wet: this was mentioned, though not defined, in [5] as the damp wall model. In our damp wall model there are two independent probabilities associated with bulk and wall occupation, respectively.

In this paper we consider directed, compact percolation near a damp wall and calculate the percolation probability exactly. We find the percolation probability, $P\left(p, p_{w}\right)$, to be

$$
P\left(p, p_{w}\right)=\frac{(2 p-1)^{2}}{p^{2}\left(p-p_{w}+p p_{w}\right)}, \quad p \geqslant p_{c},
$$

where $p_{w}$ is the probability of a site on the wall being wet, and $p$ is the probability of any other site being wet. In doing so we elucidate the crossover from the wet wall to the dry-wall critical behaviour. We note that when $p_{w}=1$, we obtain the wet wall percolation probability,

$$
P^{\text {wet }}=P(p, 1)=\frac{(2 p-1)}{p^{2}}, \quad p \geqslant p_{c},
$$

which was calculated previously in [14]. When $p_{w}=0$, we obtain the dry-wall percolation probability,

$$
P^{\mathrm{dry}}=P(p, 0)=\frac{(2 p-1)^{2}}{p^{3}}, \quad p \geqslant p_{c},
$$

which was calculated previously in [18].

Also, as a byproduct of finding the percolation probability for the damp wall case, we obtain the partition function of vesicles having two interaction parameters with a wall-see (4.69).

However, first we review how each of the cases so far considered can be mapped onto various problems of directed walks, and introduce a new mapping for the damp model. This 


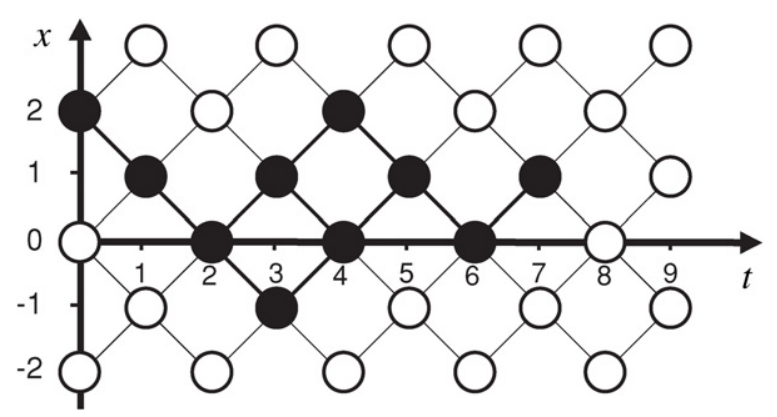

Figure 1. A directed, compact percolation cluster on the rotated square lattice. This cluster has probability $p^{7} q^{9}$.

allows comparison of the solution methods in a common framework and gives rise to an explanation of the origin of different mathematical complexities of various cases: different cases require a different number of walks and different numbers of walk parameters. We find an explicit expression for the generating function of the walk problem associated with the damp case. We note that this walk model is also related to the ASEP model: a stochastic traffic flow model. Finally, we introduce a functional-equation approach to the solution which should prove useful in the future work on these problems.

\section{Directed compact percolation in the bulk}

\subsection{The model}

Directed compact percolation can be defined through a growth process. We shall restrict ourselves to the square lattice in this work. Starting with an integer grid in coordinates $t$ (horizontal) and $x$ (vertical) we utilize a (directed) square lattice turned through $\pi / 4$ where points $(t, x)$ are sites of the grid such that $t \in \mathbb{N} \cup\{0\}, x \in \mathbb{Z}$ and $t+x$ is even, see figure 1 . Moreover, the (directed) bonds of the lattice occur between sites $(t, x)$ and $(t+1, x+1)$, and between sites $(t, x)$ and $(t+1, x-1)$.

The sites of the lattice are deemed to be 'occupied'/ 'wet' or 'not occupied'/ 'dry' by the following process. A set of $m$ contiguous sites in the column $t=0$ is fixed to be wet. In particular we can choose $(0,2),(0,4), \ldots,(0,2 m)$ to be wet. We shall concentrate on the case $m=1$ in this work. From this seed a cluster of wet sites is grown in the following way. As we see in figure 1, the cluster produced by the process described below remains contiguous, though of variable size. We label the coordinates of the wet sites in column $t$ as $\left(t, x_{j}\right)$, where $j=1, \ldots, w(t)$ and $x_{j}=b(t)+2(j-1)$. Here, $b(t)$ is therefore the $x$-coordinate of the bottom-most wet site in column $t$. We have $b(0)=2$ and $w(0)=m$.

The process proceeds a column at a time. Let the currently rightmost occupied column have the horizontal coordinate $T$. Although the order is flexible, for the sake of definiteness, start with considering the site $(T+1, b(T)-1)$. It will be occupied or not with a probability $p$. There is no need to consider sites with smaller $x$-coordinates as these will remain dry in the process. Move up the column site-by-site using the general rule that a site $(T+1, x)$ becomes wet with certainty if both sites $(T, x \pm 1)$ are wet, and with probability $p$ if only one of these sites is wet, and so stays dry with probability $q=1-p$. If both sites $(T, x \pm 1)$ are dry then the site $(T+1, x)$ remains dry. These rules ensure the contiguity of the cluster in any column, 
and hence the descriptor compact in the title of the model. The process either stops after a finite number of columns, say $\ell$ (being the length of the cluster), or continues indefinitely.

Note that as the process moves from column $T$ to $T+1$ the probability of the cluster obtains a factor of $p^{2}$ if the width of the cluster increases by $1, p q$ if the width stays unchanged and $q^{2}$ if the width decreases by 1 . Also note that the size of a cluster $s=\sum_{t} w(t)$, being the number of wet sites, is not simply related to the length of a cluster $\ell$.

Now, consider the configurations produced by this growth process and the associated probabilities. We label the set of all configurations of finite length $\ell$ by $\Omega_{\ell}^{\text {bulk }}$. The probability of a particular configuration $\varphi_{\ell} \in \Omega_{\ell}^{\text {bulk }}$ is

$$
\pi\left(\varphi_{\ell}\right)=p^{2 i}(p q)^{c} q^{2 d},
$$

where $i, c$ and $d$ are the number of times the width of the cluster increases, stays constant and decreases in width, respectively. Now we know that $i+c+d=\ell$ and since a cluster that terminates in a finite length $(w(\ell)=0)$ must decrease its width $m$ more times than it increases it, this gives $d=i+m$. Hence we have

$$
\pi^{\text {bulk }}\left(\varphi_{\ell}\right)=(p q)^{\ell-m} q^{2 m} .
$$

We define the probability that the cluster is finite as

$$
Q^{\text {bulk }}(p)=\sum_{\ell=1}^{\infty} \sum_{\varphi_{\ell} \in \Omega_{\ell}^{\text {bulk }}} \pi\left(\varphi_{\ell}\right)
$$

and so substituting our expression for the configuration probability (2.2) gives

$$
Q^{\text {bulk }}(p)=\left(\frac{q}{p}\right)^{m} \sum_{\ell=1}^{\infty}(p q)^{\ell} \sum_{\varphi_{\ell} \in \Omega_{\ell}^{\text {bulk }}} 1=\left(\frac{q}{p}\right)^{m} \sum_{\ell=1}^{\infty} b_{\ell}(p q)^{\ell},
$$

where $b_{\ell}=\left|\Omega_{\ell}\right|$.

There are several quantities of interest. The key quantity, on which we focus in this paper, is the probability $P(p)$ that an infinite cluster is grown from the seed, given by

$$
P^{\text {bulk }}(p)=1-Q^{\text {bulk }}(p),
$$

which is known as the percolation probability. Also of interest is the average length of finite clusters $\langle\ell\rangle(p)$ given by

$$
\langle\ell\rangle^{\text {bulk }}(p)=\frac{\sum_{\ell=1}^{\infty} \ell b_{\ell}(p q)^{\ell}}{\sum_{\ell=1}^{\infty} b_{\ell}(p q)^{\ell}} .
$$

The other quantity usually considered is the average size of finite clusters $\langle s\rangle^{\text {bulk }}(p)$, which needs to be defined from first principles as

$$
\langle s\rangle^{\text {bulk }}(p)=\frac{\sum_{\ell=1}^{\infty} \sum_{\varphi_{\ell} \in \Omega_{\ell}} s\left(\varphi_{\ell}\right) \pi\left(\varphi_{\ell}\right)}{\sum_{\ell=1}^{\infty} \sum_{\varphi_{\ell} \in \Omega_{\ell}} \pi\left(\varphi_{\ell}\right)} .
$$

Directed, compact percolation has been shown [8] to undergo a phase transition in a similar way to that of ordinary percolation. The order parameter is the percolation probability and there exists a critical point at some value of the occupation probability $p=p_{c}$. For $p<p_{c}$ only finite clusters are produced by the process described above and $P(p)=0$. However, for $p>p_{c}$ an infinite cluster is found to exist and $P(p)>0$. A critical exponent $\beta$ is defined by the way $P(p)$ approaches zero as $p$ approaches $p_{c}$ from above:

$$
P(p) \sim B\left(p-p_{c}\right)^{\beta}
$$




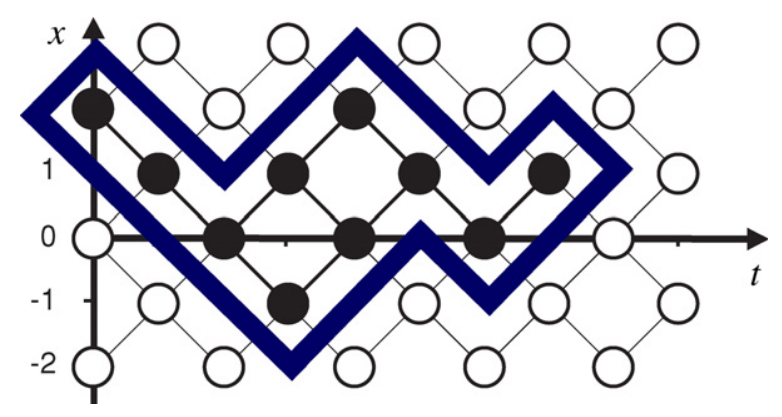

Figure 2. Mapping the cluster to a pair of directed walks, of weight $z^{9}$.

as $p \rightarrow p_{c}^{+}$. The exponent $\tau_{1}$ is defined by the behaviour of the average length $\langle\ell\rangle(p)$ near $p_{c}$,

$$
\langle\ell\rangle(p) \sim C_{ \pm}\left|p-p_{c}\right|^{-\tau_{1}} \quad \text { as } \quad p \rightarrow p_{c} .
$$

Other exponents and a scaling theory linking the exponents have been developed $[8,10]$.

We shall now confine ourselves to the case of $m=1$, where the seed is in a single occupied site.

\subsection{Mapping to directed walks}

The configurations of the directed compact percolation clusters can be placed in bijection with a problem of directed walks. This is true in both the bulk and the various wall cases discussed later. In the bulk case for $m=1$ the directed walk problem involves two fully directed walks on the square lattice that start and end at the same lattice site: that is, the problem of staircase polygons [3]. In particular the problem of finding $P(p)$ becomes equivalent to finding the perimeter generating function for staircase polygons [6]. To see this, consider the boundary of a cluster on the dual lattice (where $t+x$ is odd), see figure 2. Moving from left to right the boundary begins at $(-1,2)$ and finishes when $t=\ell$. Between each column of the cluster there are two steps of the boundary (of which there are $(\ell-1)$ such pairs) and additional pairs of boundary steps at each end of the cluster. There are $(\ell+1)$ pairs of steps in total. Given that the steps in the boundary are only those with unit vectors $(1,1)$ or $(1,-1)$ the boundary is clearly made of two fully directed walks on the square lattice which start and end at the same sites but otherwise avoid each other; these are staircase polygons. It is also simple to see that all staircase polygons of non-zero perimeter are boundaries for percolation clusters and that the mapping is one-to-one. The probability of a cluster is given by equation (2.2), with $m=1$, that is $(p q)^{\ell-1} q^{2}$ and the boundary walk of the same configuration is given weight $z^{\ell+1}$. Now consider the perimeter generating function for staircase polygons

$$
G_{\mathrm{sc}}(z)=\sum_{t=2}^{\infty} a_{t} z^{t}=\frac{1-2 z-\sqrt{1-4 z}}{2},
$$

where $z$ is associated with the half-perimeter $t$, and $a_{t}$ is the number of staircase polygons with half-perimeter $t$. The bijection described above has established that $b_{\ell}=a_{\ell+1}$ and so

$$
G_{\mathrm{sc}}(z)=z \sum_{\ell=1}^{\infty} b_{\ell} z^{\ell} .
$$

Hence by making the mapping $z=p q$ we have

$$
Q^{\text {bulk }}(p)=p^{-2} G_{\mathrm{sc}}(p q) \text {. }
$$


The average length of finite clusters $\langle\ell\rangle(p)$ can also be found from the perimeter staircase polygon generating function via equation (2.6). If we define $M_{\mathrm{sc}}(z)$ as

$$
M_{\mathrm{sc}}(z)=z \frac{\mathrm{d}}{\mathrm{d} z}\left(\log \left[\frac{G_{\mathrm{sc}}(z)}{z}\right]\right)
$$

then the mean length of finite clusters is

$$
\langle\ell\rangle^{\text {bulk }}(p)=M_{\mathrm{sc}}(p q) \text {. }
$$

The average size $\langle s\rangle(p)$, and indeed all the moments of the size distribution, can be found by consideration of the area-perimeter generating function [6].

\subsection{Solution}

The mapping above immediately gives us the percolation probability as

$$
P^{\text {bulk }}(p)= \begin{cases}0 & p<\frac{1}{2} \\ \frac{2 p-1}{p^{2}} & p \geqslant \frac{1}{2}\end{cases}
$$

This implies that there is a critical point at $p=p_{c}=\frac{1}{2}$, such that below $p_{c}$ only finite clusters are formed, whereas above $p_{c}$ there exists an infinite cluster and correspondingly $P(p)>0$. Moreover the critical exponent $\beta^{\text {bulk }}=1$ in the bulk.

The average length of finite clusters $\langle\ell\rangle(p)$ can also be found from the perimeter staircase polygon generating function (equation (2.14)) as

$$
\langle\ell\rangle^{\text {bulk }}(p)=\frac{1}{|2 p-1|}
$$

Therefore the critical exponent $\tau_{1}^{\text {bulk }}=1$ in the bulk.

\section{Directed compact percolation near a wall}

\subsection{Definition for a general 'damp' wall}

Let us consider the growth process again starting from a seed of size $m=1$, and modify it in two ways. First, to introduce a wall, nominally at $x=1$, we restrict occupation of sites to $x \geqslant 1$. Second, we introduce a separate probability of occupation, $p_{w}$, for sites on the wall in the following way. We note prior to this that sites on the wall only occur for odd values of the horizontal coordinate $t$. Consider that the growth process is currently in column $T=2 r$. If the site $(2 r, 2)$ is wet then the site $(2 r+1,1)$ is wet with probability $p_{w}$, and hence is dry with probability $q_{w}=1-p_{w}$. If the site $(2 r, 2)$ is dry then the $(2 r+1,1)$ is dry with certainty. For all other sites with $x>1$ in column $2 r+1$, and indeed for any site with $x>1$ in a column with even horizontal coordinate, the same growth rules as in the bulk case hold. All sites with $x<1$ are fixed to be dry.

To consider the probability of any finite cluster one needs to know its length $\ell$, the number of sites, $v_{1}$, with $x=1$ that are occupied and the number of sites, $v_{2}$, with $x=2$ that are occupied. Relative to the bulk probability of $(p q)^{\ell-1} q^{2}$, each occupied site at $x=2$ where there is no wet site in the subsequent column at $x=1$ (of which there are $v_{2}-v_{1}$ ) adjusts the probability by a factor of $\frac{q_{w}}{q}$. Each wet site on the wall (of which there are $v_{1}$ ) adjusts the probability in two ways: the non-occupation of $x=0$ supplies a factor of $q^{-1}$ 


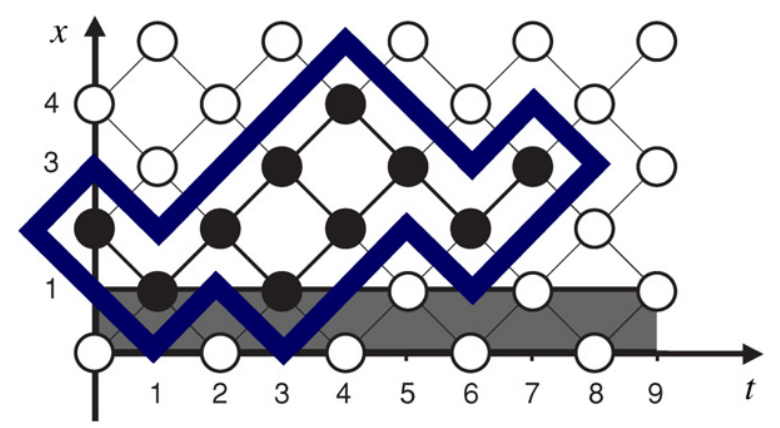

Figure 3. A cluster of a damp wall model. This cluster has probability $p^{5} q^{5} p_{w}^{2} q_{w}^{2}$.

while the different probability of occupying the site at $x=1$ gives a factor of $\frac{p_{w}}{p}$. Therefore the probability of such a configuration, $\varphi_{\ell, v_{1}, v_{2}} \in \Omega_{\ell, v_{1}, v_{2}}$, is

$$
\pi\left(\varphi_{\ell, v_{1}, v_{2}}\right)=(p q)^{\ell-1} q^{2}\left(\frac{p_{w}}{p}\right)^{v_{1}}\left(\frac{q_{w}}{q}\right)^{v_{2}-v_{1}}\left(\frac{1}{q}\right)^{v_{1}} .
$$

The probability that the growth process produces a finite cluster is

$$
Q\left(p, p_{w}\right)=\sum_{\ell=1}^{\infty} \sum_{v_{1}, v_{2}} \sum_{\varphi \in \Omega_{\ell, v_{1}, v_{2}}} \pi\left(\varphi_{\ell, v_{1}, v_{2}}\right)
$$

which can be written as

$$
Q\left(p, p_{w}\right)=\left(\frac{q}{p}\right) \sum_{\ell=1}^{\infty} \sum_{v_{1}, v_{2}} c_{\ell, v_{1}, v_{2}}\left(\frac{p_{w}}{p q_{w}}\right)^{v_{1}}\left(\frac{q_{w}}{q}\right)^{v_{2}}(p q)^{\ell},
$$

where $c_{\ell, v_{1}, v_{2}}=\left|\Omega_{\ell, v_{1}, v_{2}}\right|$.

The percolation probability is given by $P\left(p, p_{w}\right)=1-Q\left(p, p_{w}\right)$, and the mean-cluster length and other averages in an analogous way to the bulk expressions.

3.1.1. Mapping to directed walks. In the same way as in the bulk the clusters of the growth process can be put into bijection with configurations of staircase polygons. However, to accommodate the damp wall, the restriction that the wet sites must have $x \geqslant 1$ implies that the sites visited by the polygon must obey $x \geqslant 0$. Also, the weights of the polygon must be modified to allow for the different occupation probability on the wall. Let us define a particular problem of staircase polygons above a wall at $x=0$ where one end is fixed at $(-1,2)$ as in the bulk case, and the other end is free with the rest: see figure 3. Now in addition to attaching the fugacity $z$ to each pair of steps in the polygon we attached extra fugacities to steps that move from $x=1$ to $x=2$ and those that move from $x=1$ to $x=0$. Let the number of staircase polygons of half-perimeter $t^{\prime}$, with $u_{1}$ steps that move from $x=1$ to $x=0$ and $u_{2}$ steps that move from $x=1$ to $x=2$ be $d_{t^{\prime}, u_{1}, u_{2}}$. The generating function for this polygon problem, which we shall call damp staircase polygons, or damp vesicles for short, is

$$
G_{\mathrm{dv}}\left(z ; \kappa_{1}, \kappa_{2}\right)=\sum_{t^{\prime}=2} \sum_{u_{1}, u_{2}} d_{t^{\prime}, u_{1}, u_{2}} \kappa_{1}^{u_{1}} \kappa_{2}^{u_{2}} z^{t^{\prime}}
$$

Each step from $x=1$ to $x=0$ can be mapped one-to-one with wet sites at $x=1$. Hence $u_{1}=v_{1}$. Each step from $x=1$ to $x=2$ can be mapped one-to-one with certain pairs of sites 
at $x=1$ and $x=2$, precisely, a wet site at $x=2$ in one column and a dry site at $x=1$ in the next column. So $u_{2}=v_{2}-v_{1}$. Therefore, we have $c_{\ell, v_{1}, v_{2}}=d_{\ell+1, v_{1}, v_{2}-v_{1}+1}$. Hence we have

$$
G_{\mathrm{dv}}\left(z ; \kappa_{1}, \kappa_{2}\right)=z \sum_{\ell=1} \sum_{v_{1}, v_{2}} c_{\ell, v_{1}, v_{2}}\left(\frac{\kappa_{1}}{\kappa_{2}}\right)^{v_{1}} \kappa_{2}^{v_{2}} z^{\ell} .
$$

Correspondence to the percolation problem is made by the associations $\frac{\kappa_{1}}{\kappa_{2}}=\frac{p_{w}}{p q_{w}}, \kappa_{2}=\frac{q_{w}}{q}$ and $z=p q$. Therefore, making the substitutions

$$
\begin{aligned}
& z=p q \\
& \kappa_{1}=\frac{p_{w}}{p q} \\
& \kappa_{2}=\frac{q_{w}}{q}
\end{aligned}
$$

into the damp vesicle generating function $G_{\mathrm{dv}}\left(z ; \kappa_{1}, \kappa_{2}\right)$ gives us the correspondence

$$
Q\left(p, p_{w}\right)=\left(\frac{1}{p^{2}}\right) G_{\mathrm{dv}}\left(p q ; \frac{p_{w}}{p q}, \frac{q_{w}}{q}\right) .
$$

We now review the limiting cases already analysed in the literature, namely, the wet wall and the $d r y$ wall. Before we do this let us rewrite the probability of a cluster in the following way:

$$
\pi\left(\varphi_{\ell, v_{1}, v_{2}}\right)=p^{\ell-v_{1}-1} q^{\ell-v_{2}+1} p_{w}^{v_{1}} q_{w}^{v_{2}-v_{1}} .
$$

We note that $1 \leqslant v_{2}, 0 \leqslant v_{1} \leqslant v_{2}$ and $v_{1}+v_{2} \leqslant \ell$.

\subsection{Wet wall}

If we set $p_{w}=1$ and so $q_{w}=0$ then all sites on the wall are occupied, or wet, and this is known as the wet wall model. The effect of taking this limit is that the clusters remain attached to the wall. Any cluster must have as many sites wet at $x=1$ as at $x=2$. We therefore have $v_{2}=v_{1}$ and $v_{1}+v_{2}=\ell$. This implies that $\ell$ must be even so let $\ell=2 r$ and hence $v_{1}=v_{2}=r$. The probability of a cluster then becomes

$$
\pi^{\text {wet }}\left(\varphi_{\ell}\right)=(p q)^{r-1} q^{2}
$$

and the probability that a cluster is finite can be written as

$$
Q^{\mathrm{wet}}(p)=\left(\frac{q}{p}\right) \sum_{r=1}^{\infty} \hat{c}_{r}(p q)^{r},
$$

where $\hat{c}_{r}=\left|\hat{\Omega}_{r}^{\text {wet }}\right|$ and $\hat{\Omega}_{r}^{\text {wet }}$ is the set of clusters of length $r$. We note that this is a singular limit as the probability measure is zero whenever $v_{1} \neq v_{2}$.

The consequence of taking this limit $q_{w} \rightarrow 0$ is that the mapping to a directed walk problem is modified. This occurs because if one attempts to use the staircase polygons as the mapped configuration one notices that only polygons where the bottom walk is fixed in a zig-zag configuration have non-zero weight. Therefore one can use a mapping to a single walk, being the top boundary walk on the dual lattice: see figure 4 .

We consider a walk beginning at $(0,3)$, and tracing the upper boundary of the cluster of length $2 r$ in the positive $t$ direction (see figure 4). We note that the final two steps must be in the $(1,-1)$ direction. In free directed walks above a surface and finishing on the surface only the final step is fixed in such a way. Therefore we consider only the part of the boundary walk that finishes in $2 r-1$ steps at height $x=2$. We note that because of the wet condition this 


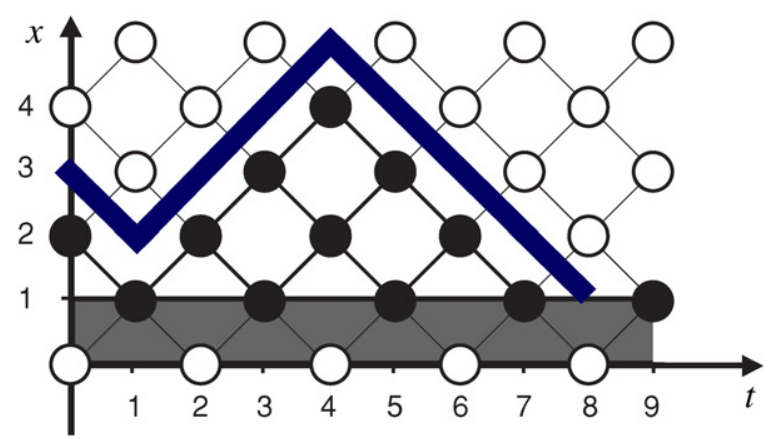

Figure 4. A cluster of a wet wall model with a boundary shown. This cluster has probability $p^{3} q^{5}$.

part of the boundary walk always obeys $x \geqslant 2$. This is then a (reversed) Ballot path [4] of length $2 r-1$ and end height of 1 . The generating function of such Ballot walks is

$$
G_{B}(y)=\sum_{r=1} \hat{d}_{r} y^{r}
$$

where the variable $y$ is associated with the half-length $r$ of the walk, and $\hat{d}_{r}=B_{2 r-1,1}$, where $B_{n, h}$ are Ballot numbers as defined in [4] (there is more than one notation in the literature). It is clear that $\hat{c}_{r}=\hat{d}_{r}$ for $r \geqslant 1$ and hence

$$
Q^{\text {wet }}(p)=\left(\frac{q}{p}\right) G_{B}(p q) .
$$

Now

$$
B_{n, h}=\frac{2(h+1)}{(n+h+2)}\left(\begin{array}{c}
n \\
\frac{n-h}{2}
\end{array}\right)
$$

so

$$
\hat{d}_{r}=B_{2 r-1,1}=\frac{2}{r+1}\left(\begin{array}{c}
2 r-1 \\
r-1
\end{array}\right)=\frac{1}{r+1}\left(\begin{array}{c}
2 r \\
r
\end{array}\right)=C_{r},
$$

where $C_{r}$ are the Catalan numbers. Hence

$$
G_{B}(y)=\sum_{r=1}^{\infty} C_{r} y^{r}
$$

Hence,

$$
G_{B}(y)=\frac{1-2 y-\sqrt{1-4 y}}{2 y} .
$$

This, in turn, implies that

$$
\begin{aligned}
Q^{\text {wet }}(p) & =\frac{1-2 p(1-p)-|2 p-1|}{2 p^{2}} \\
& = \begin{cases}1, & p \leqslant \frac{1}{2} \\
\frac{q^{2}}{p^{2}}, & p>\frac{1}{2} .\end{cases}
\end{aligned}
$$


That is, there is a critical point at $p=1 / 2$ as in the bulk model and that the order parameter exponent $\beta$ is also the same as in the bulk, namely $\beta^{\text {wet }}=1$.

The average cluster length can also be calculated from knowledge of $G_{B}(y)$ as

$$
\langle\ell\rangle^{\text {wet }}(p)=M_{D}(p q)=\frac{1}{|2 p-1|},
$$

where

$$
M_{D}(y)=2 y \frac{\mathrm{d}}{\mathrm{d} y}\left(\log G_{B}(y)\right)=\frac{1}{\sqrt{1-4 y}} .
$$

We note that the mean length of finite clusters for a wet wall is identical to that of the bulk case and so the exponent $\tau_{1}^{\text {wet }}=1$ in the wet model.

\subsection{Dry wall}

If we set $p_{w}=0$, and so $q_{w}=1$, then all sites on the wall are unoccupied (and unable to become occupied), or dry, and we have the dry-wall model. We now have $v_{1}=0$ and the probability of a cluster of length $\ell$ with $v_{2}$ wet sites at $x=2$ is

$$
\pi^{\mathrm{dry}}\left(\varphi_{\ell, v_{2}}\right)=p^{\ell-1} q^{\ell-v_{2}+1}=\frac{q}{p}(p q)^{\ell}\left(\frac{1}{q}\right)^{v_{2}}
$$

and the probability that a cluster is finite can be written as

$$
Q^{\mathrm{dry}}(p)=\left(\frac{q}{p}\right) \sum_{\ell=1}^{\infty} \sum_{v_{2}} \check{c}_{\ell, v_{2}}\left(\frac{1}{q}\right)^{v_{2}}(p q)^{\ell},
$$

where $\check{c}_{\ell, v_{2}}=\left|\Omega_{\ell, v_{2}}^{\mathrm{dry}}\right|$ and $\Omega_{\ell, v_{2}}^{\mathrm{dry}}$ is the set of all clusters of length $\ell$ with $v_{2}$ wet sites at $x=2$.

Since dry wall is the limit $p_{w} \rightarrow 0$ one can use the mapping described above for the damp wall model onto the staircase polygons with the two wall weights $\kappa_{1}$ and $\kappa_{2}$ where $\kappa_{1} \rightarrow 0$. This means that there are no steps of the lower boundary of the polygon between $x=1$ and $x=0$ and so consequently no sites of that walk at $x=0$. Essentially the cluster configurations now map one-to-one with staircase polygons starting at $(-1,2)$ and not going below $x=1$ (as opposed to $x=0$ as in the full damp case). Consider the generating function, $G_{r v}$, for such a staircase vesicle (polygons) made up of two directed walks starting at $(-1,2)$ that mutually avoid except at this first site and also the final site, picking up the weight $z^{t^{\ell+1}} \kappa_{2}^{v_{2}}$ with $\ell$ and $v_{2}$ defined as previously. We have

$$
G_{r v}\left(z ; \kappa_{2}\right)=G_{\mathrm{dv}}\left(z ; 0, \kappa_{2}\right)
$$

and

$$
Q^{\text {dry }}(p)=p^{-2} G_{r v}\left(p q, \frac{1}{q}\right) .
$$

An expression for the generating function was found in [5],

$$
\begin{gathered}
G_{r v}(z ; \kappa)=\frac{z^{2} \kappa(\kappa-2)}{(\kappa-1)^{2}}\left[1+\left(1+\frac{\omega}{z}\right)\left(\frac{\omega-2 z^{2}-\sqrt{\omega\left(\omega-4 z^{2}\right)}}{2 z^{2}}\right)\right] \theta(\kappa-2) \\
+\frac{z^{2}}{\kappa-1} \sum_{r=0}^{\infty} z^{2 r}\left(C_{r}+z C_{r+1}\right) \sum_{s=r+1}^{\infty} C_{s} \omega^{s-r},
\end{gathered}
$$


where $\omega=\frac{\kappa-1}{\kappa^{2}}$. This gives an expression for the dry case percolation probability, $P^{\mathrm{dry}}(p)=1-Q^{\mathrm{dry}}(p)$, as

$$
P^{\text {dry }}(p)= \begin{cases}0, & p \leqslant \frac{1}{2} \\ \frac{(2 p-1)^{2}}{p^{3}} & p>\frac{1}{2},\end{cases}
$$

which immediately gives us that $\beta^{\text {dry }}=2$.

In [11], differential approximants were used to find a differential equation satisfied by the unnormalized mean length $\bar{L}(p)$ :

$$
\begin{aligned}
p^{2}(1-p)(1- & 2 p)\left(1+4 p-4 p^{2}\right) \frac{\mathrm{d}^{2} \bar{L}}{\mathrm{~d} p^{2}}+p\left(7-8 p-46 p^{2}+72 p^{3}-24 p^{4}\right) \frac{\mathrm{d} \bar{L}}{\mathrm{~d} p} \\
& +\left(9-28 p-2 p^{2}+24 p^{3}-8 p^{4}\right) \bar{L}=9-12 p+12 p^{2}
\end{aligned}
$$

This was solved numerically in [11] with the result

$$
\bar{L}(p) \cong B^{ \pm} \log |2 p-1|+C^{ \pm}
$$

as $p \rightarrow 1 / 2$, where $B^{-}=B^{+}=-2.547, C^{-}=4.097$ and $C^{+}=-3.901$. This implies that, effectively, $\tau_{1}^{\text {dry }}=0$. It was conjectured that $B^{-}=B^{+}=-\frac{8}{\pi}$ and $C^{-}-C^{+}=8$. These conjectures were confirmed by the exact results of [5] with $C^{-}=\frac{\log 8-8}{\pi}+4=4.101148 \ldots$ which differs from the numerical calculation by one part in a thousand.

In [5], Zeilberger's algorithm is used to obtain an exact expression for the unnormalized mean length,

$$
\begin{aligned}
\bar{L}(p)=\frac{1}{8 p^{3}} & \left(-5+4 z+6 \sqrt{1-4 z}-\frac{8 E\left(16 z^{2}\right)}{\pi}+\frac{2(3-4 z)(1+4 z) K\left(16 z^{2}\right)}{\pi}\right) \\
& +\theta\left(p-p_{c}\right) \frac{(1-p)(3-2 p)}{p^{3}},
\end{aligned}
$$

where $z=p(1-p)$.

Also in [5], the asymptotic form for $\bar{S}(p)$, the unnormalized mean size of clusters, is found,

$$
\bar{S}(p) \cong \frac{1}{2 p-1}\left\{A^{-}+B^{-}(2 p-1)^{4} \log |2 p-1|\right\},
$$

where $A^{-}=\frac{32}{3 \pi}-\frac{1}{2}$ and $B^{-}=\frac{8}{\pi}$, confirming the estimate for $A^{-}$in [11] to be very accurate, only differing from the exact value by 1 in the sixth decimal place.

\section{Solution for the general damp case}

Let us recall that to find the percolation probability $P\left(p, p_{w}\right)$ one can instead calculate the generating function, described above, for damp vesicles $G_{\mathrm{dv}}\left(z ; \kappa_{1}, \kappa_{2}\right)$, and then use the correspondence (3.9). Essentially then the generating variable $z$ becomes dependent on the parameters $\kappa_{1}$ and $\kappa_{2}$. We refer to these values as the percolation values. We shall first consider the calculation of the generating function $G_{\mathrm{dv}}\left(z ; \kappa_{1}, \kappa_{2}\right)$ for independent values of the generating variable $z$. 


\subsection{Generating function of the damp vesicle problem}

We will find the generating function $G_{\mathrm{dv}}\left(z ; \kappa_{1}, \kappa_{2}\right)$ by considering associated partition functions. Let us denote the finite perimeter partition functions for staircase vesicles (polygons) with one end free as $Z_{t^{\prime}}^{v}\left(\kappa_{1}, \kappa_{2}\right)$, so that

$$
Z_{t^{\prime}}^{v}\left(\kappa_{1}, \kappa_{2}\right)=\sum_{u_{1}, u_{2}} d_{t^{\prime}, u_{1}, u_{2}} \kappa_{1}^{u_{1}} \kappa_{2}^{u_{2}}
$$

Here the half-perimeter is $t^{\prime}$. Therefore,

$$
G_{\mathrm{dv}}\left(z ; \kappa_{1}, \kappa_{2}\right)=\sum_{t^{\prime} \geqslant 2} Z_{t^{\prime}}^{v}\left(\kappa_{1}, \kappa_{2}\right) z^{t^{\prime}}
$$

Let us define the partition function, $Z_{t}^{\mathcal{T}}\left(x \mid 1 ; \kappa_{1}, \kappa_{2}\right)$, for weighted pairs of mutually avoiding directed walks of length $t$, weighted with $\kappa_{1}$ for each step from $x=1$ to $x=0$, and with $\kappa_{2}$ for each step from $x=1$ to $x=2$, beginning at $(0,1)$ and $(0,3)$ and ending at particular points $(t, x)$ and $(t, x+2)$, respectively. However, let us include an extra factor of $\kappa_{2}$ if $x=1$ : this takes care of the weight of a final step of the vesicle if it is between $x=1$ and $x=2$. Hence,

$$
Z_{t+2}^{v}\left(\kappa_{1}, \kappa_{2}\right)=\sum_{x=0}^{t+1} Z_{t}^{\mathcal{T}}\left(x \mid 1 ; \kappa_{1}, \kappa_{2}\right) .
$$

The mathematical reason for this peculiar definition will become clear below. In any case we now have

$$
G_{\mathrm{dv}}\left(z ; \kappa_{1}, \kappa_{2}\right)=z^{2} \sum_{t \geqslant 0} z^{t} \sum_{x=0}^{t+1} Z_{t}^{\mathcal{T}}\left(x \mid 1 ; \kappa_{1}, \kappa_{2}\right) .
$$

If we define the generating function $G\left(z ; \kappa_{1}, \kappa_{2}\right)$ simply as

$$
G\left(z ; \kappa_{1}, \kappa_{2}\right)=z^{-2} G_{\mathrm{dv}}\left(z ; \kappa_{1}, \kappa_{2}\right)=\sum_{t \geqslant 0} z^{t} \sum_{x=0}^{t+1} Z_{t}^{\mathcal{T}}\left(x \mid 1 ; \kappa_{1}, \kappa_{2}\right),
$$

where we note that the first few terms of the generating function are

$G\left(z ; \kappa_{1}, \kappa_{2}\right)=\kappa_{2}+\left(\kappa_{1}+\kappa_{2}\right) z+\left(\kappa_{2}+2 \kappa_{1} \kappa_{2}+\kappa_{2}^{2}\right) z^{2}+\left(2 \kappa_{1}^{2}+2 \kappa_{2}+4 \kappa_{1} \kappa_{2}+2 \kappa_{2}^{2}\right) z^{3}+\cdots$,

then

$$
P\left(p, p_{w}\right)=1-q^{2} G\left(p q ; \frac{p_{w}}{p q}, \frac{q_{w}}{q}\right) .
$$

To begin we call upon the Gessel-Viennot determinant $[15,16]$ to express this fixed-end vesicle partition function in terms of single-walk partition functions as

$$
Z_{t}^{\mathcal{T}}\left(x \mid 1 ; \kappa_{1}, \kappa_{2}\right)=\left|\begin{array}{ll}
Z_{t}^{s}\left(x \mid 1 ; \kappa_{1}, \kappa_{2}\right) & Z_{t}^{s}\left(x+2 \mid 1 ; \kappa_{1}, \kappa_{2}\right) \\
Z_{t}^{s}\left(x \mid 3 ; \kappa_{1}, \kappa_{2}\right) & Z_{t}^{s}\left(x+2 \mid 3 ; \kappa_{1}, \kappa_{2}\right)
\end{array}\right|,
$$

where $Z_{t}^{s}\left(x_{f} \mid x_{i} ; \kappa_{1}, \kappa_{2}\right)$ is the partition function for single walks beginning at $x=x_{i} \geqslant 0$ and terminating at $x=x_{f} \geqslant 0$, after $t$ steps, weighted with $\kappa_{1}$ for each step from $x=1$ to $x=0$, and with $\kappa_{2}$ for each step from $x=1$ to $x=2$ and with the extra weight $\kappa_{2}$ if $x_{f}=1$ attached to the final site of the walk. We shall refer to these unusually weighted walks as damp walks. What is important to note is that one could equivalently weight each step from $x=0$ to $x=1$ with the weight $\kappa_{1}$ and each step from $x=2$ to $x=1$ with weight $\kappa_{2}$ and add the additional weight $\kappa_{2}$ if $x_{i}=1$ to obtain the same partition function. This implies the symmetry

$$
Z_{t}^{s}\left(x_{f} \mid x_{i} ; \kappa_{1}, \kappa_{2}\right)=Z_{t}^{s}\left(x_{i} \mid x_{f} ; \kappa_{1}, \kappa_{2}\right)
$$


and it is this symmetry that plays an important role in using the constant term method to solve for $Z_{t}^{s}$. Of course, from equations (4.5) and (4.8) it is precisely the single-walk partition function that we are required to find.

It is therefore apropos to consider the mathematics of the solution for the single-walk partition function $Z_{t}^{s}\left(x_{f} \mid x_{i} ; \kappa_{1}, \kappa_{2}\right)$. Importantly as well as being able to write an explicit solution for $Z_{t}^{s}$ one can also find a recurrence relation in $t$ for it that allows one to derive a recurrence relation for $Z_{t}^{\mathcal{T}}$. The recurrence can be solved using generating functions which is precisely the required result.

4.1.1. Single damp walk partition function. Because of the symmetry noted above let us consider $Z_{t}^{s}\left(x_{f} \mid x_{i} ; \kappa_{1}, \kappa_{2}\right)$ to be that partition function for a single directed walk of length $t$ beginning at $\left(0, x_{i}\right)$ and ending at $\left(t, x_{f}\right)$, weighted with $\kappa_{1}$ for each step from $x=0$ to $x=1$, and with $\kappa_{2}$ for each step from $x=2$ to $x=1$, with an extra weight $\kappa_{2}$ if $x_{i}=1$, and constrained such that $x \geqslant 0$.

First we consider the constraints due to the shape of the lattice-as the walk can only pass through points such that $x+t$ is odd. So for $t=1$, regardless of $x_{i}$ (where $x_{i}$ is assumed to take odd values due to the lattice we are using), we cannot have $x_{f}=1$, due to the location of sites on the lattice. Hence,

$$
Z_{1}^{s}\left(1 \mid x_{i} ; \kappa_{1}, \kappa_{2}\right)=0 .
$$

We consider a walk of trivial length. For $t=0$, the only physical possibility is if $x_{f}=x_{i}$. This is weighted by 1 for $x_{i}>1$, and by $\kappa_{2}$ if $x_{i}=1$. So we have

$$
\begin{aligned}
& Z_{0}^{s}\left(x \mid x_{i} ; \kappa_{1}, \kappa_{2}\right)=\delta_{x, x_{i}}, \quad \text { for } \quad x>1 \\
& Z_{0}^{s}\left(1 \mid x_{i} ; \kappa_{1}, \kappa_{2}\right)=\kappa_{2} \delta_{1, x_{i}} .
\end{aligned}
$$

We can combine (4.11) and (4.12) to obtain

$$
Z_{0}^{s}\left(x \mid x_{i} ; \kappa_{1}, \kappa_{2}\right)=\delta_{x, x_{i}}\left(1+\left(\kappa_{2}-1\right) \delta_{x_{i}, 1}\right) .
$$

Due to the location of the wall, we know that if a walk is at $x=0$ after $t$ steps, then after $(t-1)$ steps it was at $x=1$, with an adjustment of the weightings, so we have

$$
Z_{t}^{s}\left(0 \mid x_{i} ; \kappa_{1}, \kappa_{2}\right)=\frac{\kappa_{1}}{\kappa_{2}} Z_{t-1}^{s}\left(1 \mid x_{i} ; \kappa_{1}, \kappa_{2}\right), \quad t>0
$$

Considering walks that are at $x=1$ after $t$ steps we have

$$
Z_{t}^{s}\left(1 \mid x_{i} ; \kappa_{1}, \kappa_{2}\right)=\kappa_{2} Z_{t-1}^{s}\left(0 \mid x_{i} ; \kappa_{1}, \kappa_{2}\right)+\kappa_{2} Z_{t-1}^{s}\left(2 \mid x_{i} ; \kappa_{1}, \kappa_{2}\right) .
$$

Away from the wall, we have the general recurrence, for $t>0, x \geqslant 2$,

$$
Z_{t}^{s}\left(x \mid x_{i} ; \kappa_{1}, \kappa_{2}\right)=Z_{t-1}^{s}\left(x-1 \mid x_{i} ; \kappa_{1}, \kappa_{2}\right)+Z_{t-1}^{s}\left(x+1 \mid x_{i} ; \kappa_{1}, \kappa_{2}\right) .
$$

We list a few values of the single-walk partition function:

$$
\begin{aligned}
& Z_{0}^{s}\left(1 \mid 1 ; \kappa_{1}, \kappa_{2}\right)=\kappa_{2} \\
& Z_{2}^{s}\left(1 \mid 1 ; \kappa_{1}, \kappa_{2}\right)=\kappa_{1} \kappa_{2}+\kappa_{2}^{2} \\
& Z_{4}^{s}\left(1 \mid 1 ; \kappa_{1}, \kappa_{2}\right)=\kappa_{1}^{2} \kappa_{2}+\kappa_{2}^{2}+\kappa_{2}^{3}+2 \kappa_{1} \kappa_{2}^{2} \\
& Z_{0}^{s}\left(3 \mid 1 ; \kappa_{1}, \kappa_{2}\right)=0 \\
& Z_{2}^{s}\left(3 \mid 1 ; \kappa_{1}, \kappa_{2}\right)=\kappa_{2} \\
& Z_{4}^{s}\left(3 \mid 1 ; \kappa_{1}, \kappa_{2}\right)=2 \kappa_{2}+\kappa_{1} \kappa_{2}+\kappa_{2}^{2} .
\end{aligned}
$$


After applying a standard separation of variables ansatz $\Lambda^{t} y^{x}$ we find that equation (4.16) is satisfied by

$$
f\left(x, x_{i}, y\right)=A\left(x_{i}, y\right)(y+\bar{y})^{t}\left(y^{x}-S(y) \bar{y}^{x}\right),
$$

where $\bar{y}=y^{-1}$. For $f\left(x, x_{i}, y\right)$ to also satisfy (4.14) and (4.15), we have

$$
\Lambda^{2}(y-S(y) \bar{y})=\kappa_{1}(y-S(y) \bar{y})+\kappa_{2} \Lambda\left(y^{2}-S(y) \bar{y}^{2}\right),
$$

where $\Lambda=y+\bar{y}$. Hence,

$$
S(y)=\frac{\left(\Lambda^{2}-\kappa_{1}\right) y-\kappa_{2} \Lambda y^{2}}{\left(\Lambda^{2}-\kappa_{1}\right) \bar{y}-\kappa_{2} \Lambda \bar{y}^{2}} .
$$

The choice for $A\left(x_{i}, y\right)$ is made to ensure that the interchange $x_{i} \leftrightarrow x_{f}$ symmetry noted in equation (4.9) is obeyed:

$$
A\left(x_{i}, y\right)=\frac{1}{2}\left(\bar{y}^{x_{i}}-S(\bar{y}) y^{x_{i}}\right) .
$$

This gives, on noting that $S(\bar{y})=S(y)^{-1}$,

$$
f\left(x, x_{i}, y\right)=\frac{1}{2} \Lambda^{t}\left(y^{x-x_{i}}+y^{x_{i}-x}-S(y) \bar{y}^{x+x_{i}}-S(\bar{y}) y^{x+x_{i}}\right) .
$$

It will prove useful to write the partition function as a constant term expression: the operator $C T_{y}[]$ is defined to act on Laurent series in $y$ with finitely many negative powers, giving the coefficient of the constant term. Expanding in powers of $y$ we have

$$
y^{2} S(\bar{y})=1-\kappa_{2}+\kappa_{2}\left(2-\kappa_{1}-\kappa_{2}\right) y^{2}+O\left(y^{4}\right)
$$

and similarly expanding $\bar{y}^{2} S(y)$ in powers of $\bar{y}$, we see that $C T_{y}\left[f\left(x, x_{i}, y\right)\right]$ satisfies the boundary and initial conditions. As interchanging $y$ and $\bar{y}$ in any term under the $C T$ operation has no effect, we can hence write

$$
Z_{t}^{s}\left(x \mid x_{i} ; \kappa_{1}, \kappa_{2}\right)=C T_{y}\left[\Lambda^{t}\left(y^{x-x_{i}}-y^{2} S(\bar{y}) y^{x+x_{i}-2}\right],\right.
$$

where $y^{2} S(\bar{y})$ is to be expanded in powers of $y$.

If we define

$$
T(y)=1-y^{2} S(\bar{y})=\frac{\kappa_{2}\left(1-y^{4}\right)}{1-\left(\kappa_{1}+\kappa_{2}-2\right) y^{2}-\left(\kappa_{2}-1\right) y^{4}}
$$

then we have

$$
Z_{t}^{s}\left(x \mid x_{i} ; \kappa_{1}, \kappa_{2}\right)=C T_{y}\left[\Lambda^{t}\left(y^{x-x_{i}}-(1-T(y)) y^{x+x_{i}-2}\right)\right]
$$

and for $x_{i}=1$ we have the simple result,

$$
Z_{t}^{s}\left(x \mid 1 ; \kappa_{1}, \kappa_{2}\right)=C T_{y}\left[\Lambda^{t} T(y) y^{x-1}\right]
$$

Factorizing the denominator of $T(y)$ in $(4.30)$ as $\left(1-c y^{2}\right)\left(1-d y^{2}\right)$, we have the variable transformations

$$
c+d=\kappa_{1}+\kappa_{2}-2, \quad c d=1-\kappa_{2},
$$

that is,

$$
\kappa_{1}=(c+1)(d+1), \quad \kappa_{2}=1-c d .
$$

This gives

$$
Z_{t}^{s}\left(x \mid 1 ; \kappa_{1}, \kappa_{2}\right)=C T_{y}\left[\frac{\kappa_{2} \Lambda^{t}\left(1-y^{4}\right) y^{x-1}}{\left(1-c y^{2}\right)\left(1-d y^{2}\right)}\right] .
$$

We note that, for $c \neq d$

$$
\frac{1}{\left(1-c y^{2}\right)\left(1-d y^{2}\right)}=\frac{1}{c-d}\left(\frac{c}{1-c y^{2}}-\frac{d}{1-d y^{2}}\right) .
$$


So we have

$$
Z_{t}^{s}\left(x \mid 1 ; \kappa_{1}, \kappa_{2}\right)=C T\left[\frac{\kappa_{2} \Lambda^{t+1}\left(1-y^{2}\right) y^{x}}{c-d}\left(\frac{c}{1-c y^{2}}-\frac{d}{1-d y^{2}}\right)\right] .
$$

The single-walk partition function for the dry-wall case $U_{t}(x ; c)=Z_{t}^{s}\left(x \mid 1 ; \kappa_{1}, 1\right)$ found in Brak and Essam [5] as

$$
U_{t}(x ; c)=C T\left[\frac{\Lambda^{t} y^{x}\left(1-y^{2}\right)}{1-c y^{2}}\right]
$$

allows a neat expression for the damp case. When $x=1$,

$$
U_{t}(1 ; c)=\frac{1}{1+c} U_{t+1}(0 ; c)
$$

and so we can express $Z_{t}^{s}\left(1 \mid 1 ; \kappa_{1}, \kappa_{2}\right)$ in terms of this dry-wall partition function,

$$
Z_{t}^{s}\left(1 \mid 1 ; \kappa_{1}, \kappa_{2}\right)=\frac{\kappa_{2}}{c-d}\left(\frac{c}{1+c} U_{t+2}(0 ; c)-\frac{d}{1+d} U_{t+2}(0 ; d)\right) .
$$

Let us introduce the variables $\omega_{c}$ and $\omega_{d}$ as

$$
\omega_{c}=\frac{c}{(1+c)^{2}}, \quad \omega_{d}=\frac{d}{(1+d)^{2}} .
$$

Now from Brak and Essam [5] we know that

$$
U_{2 r+2}(0 ; c)=(1+c)\left(\omega_{c}^{-r} \frac{(c+1)(c-1)}{c^{2}} \theta(c-1)+\frac{1}{c} \sum_{s=r+1}^{\infty} C_{s} \omega_{c}^{s-r}\right)
$$

with $\theta$ being the unit step function, and so we have a complete expression for the single-walk partition function.

4.1.2. Recurrence relations for single-walk partition function. If we express $T(y)$ as

$$
T(y)=\kappa_{2} \Lambda y\left(1-y^{2}\right)+\frac{\kappa_{2} \Lambda y\left(1-y^{2}\right)\left((c+d) y^{2}-c d y^{4}\right)}{\left(1-c y^{2}\right)\left(1-d y^{2}\right)}
$$

then substituting this into (4.32) gives a recurrence in the end point position,

$Z_{t}^{s}\left(x \mid 1 ; \kappa_{1}, \kappa_{2}\right)=\kappa_{2} B_{t+1, x}+(c+d) Z_{t}^{s}\left(x+2 \mid 1 ; \kappa_{1}, \kappa_{2}\right)-c d Z_{t}^{s}\left(x+4 \mid 1 ; \kappa_{1}, \kappa_{2}\right)$,

where $B_{t, x}$ is the Ballot number,

$$
B_{t, x}=\frac{(x+1) t !}{\left(\frac{1}{2}(t+x)+1\right) !\left(\frac{1}{2}(t-x)\right) !}=C T_{y}\left[(y+\bar{y})^{t}\left(1-y^{2}\right) y^{x}\right] .
$$

Similarly,

$$
Z_{t}^{s}\left(x \mid 1 ; \kappa_{1}, \kappa_{2}\right)=\frac{\kappa_{2}\left(\omega_{c}-\omega_{d}\right)}{c-d} C T_{y}\left[\frac{\Lambda^{t+2}\left(1-y^{2}\right)}{\left(1-\omega_{c} \Lambda^{2}\right)\left(1-\omega_{d} \Lambda^{2}\right)}\right]
$$

gives a recurrence in the length $t$,

$Z_{t}^{s}\left(1 \mid 1 ; \kappa_{1}, \kappa_{2}\right)=\frac{\kappa_{2}\left(\omega_{c}-\omega_{d}\right)}{c-d} C_{\frac{1}{2} t+1}+\left(\omega_{c}+\omega_{d}\right) Z_{t+2}^{s}\left(1 \mid 1 ; \kappa_{1}, \kappa_{2}\right)-\omega_{c} \omega_{d} Z_{t+4}^{s}\left(1 \mid 1 ; \kappa_{1}, \kappa_{2}\right)$. 
4.1.3. Recurrence for partition functions for vesicles with a fixed end. We can simplify the determinant (4.8) to be only in terms of single-walk partition functions beginning at 1 , by using the relationship,

$$
Z_{t}^{s}\left(x \mid 3 ; \kappa_{1}, \kappa_{2}\right)=\frac{1}{\kappa_{2}}\left(Z_{t+2}^{s}\left(x \mid 1 ; \kappa_{1}, \kappa_{2}\right)-\left(\kappa_{1}+\kappa_{2}\right) Z_{t}^{s}\left(x \mid 1 ; \kappa_{1}, \kappa_{2}\right)\right),
$$

obtained by the general recurrences (4.14), (4.15) and (4.16). Applying this to (4.8) gives

$$
Z_{t}^{\mathcal{T}}\left(x \mid 1 ; \kappa_{1}, \kappa_{2}\right)=\frac{1}{\kappa_{2}}\left|\begin{array}{ll}
Z_{t}^{s}\left(x \mid 1 ; \kappa_{1}, \kappa_{2}\right) & Z_{t}^{s}\left(x+2 \mid 1 ; \kappa_{1}, \kappa_{2}\right) \\
Z_{t+2}^{s}\left(x \mid 1 ; \kappa_{1}, \kappa_{2}\right) & Z_{t+2}^{s}\left(x+2 \mid 1 ; \kappa_{1}, \kappa_{2}\right)
\end{array}\right| .
$$

We shall see below that we will only need to consider $Z_{t}^{\mathcal{T}}\left(1 \mid 1 ; \kappa_{1}, \kappa_{2}\right)$ to evaluate $Z_{t}^{v}\left(\kappa_{1}, \kappa_{2}\right)$ so from (4.49), if we set $x=1$ we have

$$
Z_{t}^{\mathcal{T}}\left(1 \mid 1 ; \kappa_{1}, \kappa_{2}\right)=\frac{1}{\kappa_{2}}\left|\begin{array}{ll}
Z_{t}^{s}\left(1 \mid 1 ; \kappa_{1}, \kappa_{2}\right) & Z_{t}^{s}\left(3 \mid 1 ; \kappa_{1}, \kappa_{2}\right) \\
Z_{t+2}^{s}\left(1 \mid 1 ; \kappa_{1}, \kappa_{2}\right) & Z_{t+2}^{s}\left(3 \mid 1 ; \kappa_{1}, \kappa_{2}\right)
\end{array}\right| .
$$

Applying the physical property of (4.48) (traversed backwards in this case), we have

$$
Z_{t}^{\mathcal{T}}\left(1 \mid 1 ; \kappa_{1}, \kappa_{2}\right)=\frac{1}{\kappa_{2}^{2}}\left|\begin{array}{ll}
Z_{t}^{s}\left(1 \mid 1 ; \kappa_{1}, \kappa_{2}\right) & Z_{t+2}^{s}\left(1 \mid 1 ; \kappa_{1}, \kappa_{2}\right) \\
Z_{t+2}^{s}\left(1 \mid 1 ; \kappa_{1}, \kappa_{2}\right) & Z_{t+4}^{s}\left(1 \mid 1 ; \kappa_{1}, \kappa_{2}\right)
\end{array}\right| .
$$

Now, setting $t=2 r$ and substituting into the recurrence (4.47), we have

$$
Z_{2 r}^{\mathcal{T}}\left(1 \mid 1 ; \kappa_{1}, \kappa_{2}\right)=\frac{\omega_{c}-\omega_{d}}{\kappa_{2}(c-d)}\left|\begin{array}{ll}
C_{r+1} & Z_{2 r+2}^{s}\left(1 \mid 1 ; \kappa_{1}, \kappa_{2}\right) \\
C_{r+2} & Z_{2 r+4}^{s}\left(1 \mid 1 ; \kappa_{1}, \kappa_{2}\right)
\end{array}\right|+\omega_{c} \omega_{d} Z_{2 r+2}^{\mathcal{T}}\left(1 \mid 1 ; \kappa_{1}, \kappa_{2}\right) .
$$

This will be the main recurrence that we will be required to solve.

4.1.4. Simplifying the partition function for vesicles with a free end. We now seek an expression for the free-end vesicle partition function $Z_{t}^{v}\left(\kappa_{1}, \kappa_{2}\right)$. We begin by combining a recurrence found earlier from the single-walk partition function with the Gessel-Viennot expression of the fixed-end vesicle partition function. That is, we can use (4.44) to simplify (4.49) to

$$
\begin{aligned}
& Z_{t}^{\mathcal{T}}\left(x \mid 1 ; \kappa_{1}, \kappa_{2}\right)=D_{t}(x)+\left(1-\kappa_{2}\right) Z_{t}^{\mathcal{T}}\left(x+2 \mid 1 ; \kappa_{1}, \kappa_{2}\right), \quad x \neq 0 \\
& Z_{t}^{\mathcal{T}}\left(0 \mid 1 ; \kappa_{1}, \kappa_{2}\right)=\frac{1}{\kappa_{2}}\left(D_{t}(0)+\left(1-\kappa_{2}\right) Z_{t}^{\mathcal{T}}\left(2 \mid 1 ; \kappa_{1}, \kappa_{2}\right)\right),
\end{aligned}
$$

where

$$
D_{t}(x)=\left|\begin{array}{ll}
B_{t+1, x} & Z_{t}^{s}\left(x+2 \mid 1 ; \kappa_{1}, \kappa_{2}\right) \\
B_{t+3, x} & Z_{t+2}^{s}\left(x+2 \mid 1 ; \kappa_{1}, \kappa_{2}\right)
\end{array}\right| .
$$

Using (4.32) and (4.45), we can hence write

$$
D_{t}(x)=C T_{y_{1}, y_{2}}\left[\Lambda_{1}^{t+1} \Lambda_{2}^{t}\left(\Lambda_{2}^{2}-\Lambda_{1}^{2}\right)\left(y_{1} y_{2}\right)^{x}\left(1-y_{1}^{2}\right) y_{2} T\left(y_{2}\right)\right] .
$$

Summing (4.53) over $x$ and using (4.54) gives

$$
\dot{Z}_{t}^{v}\left(\kappa_{1}, \kappa_{2}\right)=\frac{1}{\kappa_{2}}\left(\sum_{x=0}^{t+1} D_{t}(x)-c d Z_{t}^{\mathcal{T}}\left(1 \mid 1 ; \kappa_{1}, \kappa_{2}\right)\right) .
$$

We now consider separately the free-end vesicle partition function for vesicles of even length. We note that for even $t, Z_{t}^{\mathcal{T}}\left(x \mid 1 ; \kappa_{1}, \kappa_{2}\right)=0$ when $x$ is even, and also

$$
\left(\Lambda_{2}^{2}-\Lambda_{1}^{2}\right) \sum_{y=0}^{\infty}\left(y_{1} y_{2}\right)^{2 y+2}=y_{1}^{2}-y_{2}^{2}
$$


and also that $Z_{t}^{\mathcal{T}}\left(x \mid 1 ; \kappa_{1}, \kappa_{2}\right)=0$ when $x+t$ is even. So, applying these to (4.56) for $t$ even, we have

$$
\begin{aligned}
\sum_{y=0}^{r} D_{2 r}(2 y+1) & =C T_{y_{1}, y_{2}}\left[\Lambda_{1}^{2 r+1} \Lambda_{2}^{2 r}\left(y_{1}^{2}-y_{2}^{2}\right) \overline{y_{1}}\left(1-y_{1}^{2}\right) T\left(y_{2}\right)\right] \\
& =C T_{y_{1}, y_{2}}\left[\Lambda_{1}^{2 r+1} \Lambda_{2}^{2 r}\left(\left(y_{1}+\overline{y_{1}}\right)\left(1-y_{1}^{2}\right)+\left(y_{1}-\overline{y_{1}}\right)\left(1+y_{2}^{2}\right)\right) T\left(y_{2}\right)\right] .
\end{aligned}
$$

But

$C T_{y_{1}, y_{2}}\left[\Lambda_{1}^{2 r+1}\left(y_{1}-\overline{y_{1}}\right) F\left(y_{2}\right)\right]=C T_{y_{1}, y_{2}}\left[y_{1} \Lambda_{1}^{2 r+1} F\left(y_{2}\right)-\bar{y}_{1} \Lambda_{1}^{2 r+1} F\left(y_{2}\right)\right]=0$

as the second term is identical to the first when $y_{1}$ and $\overline{y_{1}}$ are interchanged. So we have

$$
\begin{aligned}
\sum_{y=0}^{r} D_{2 r}(2 y+1) & =C T_{y_{1}, y_{2}}\left[\Lambda_{1}^{2 r+2}\left(1-y_{1}^{2}\right)\right] C T_{y_{1}, y_{2}}\left[\Lambda_{2}^{2 r} T\left(y_{2}\right)\right] \\
& =C_{r+1} Z_{2 r}^{s}\left(1 \mid 1 ; \kappa_{1}, \kappa_{2}\right) .
\end{aligned}
$$

So we have an expression for the free-end partition function for vesicles of even length,

$$
Z_{2 r}^{v}\left(\kappa_{1}, \kappa_{2}\right)=\frac{1}{\kappa_{2}}\left(C_{r+1} Z_{2 r}^{s}\left(1 \mid 1 ; \kappa_{1}, \kappa_{2}\right)+\left(\kappa_{2}-1\right) Z_{2 r}^{\mathcal{T}}\left(1 \mid 1 ; \kappa_{1}, \kappa_{2}\right)\right) .
$$

We obtain a similar result for $t$ odd,

$$
\dot{Z}_{2 r+1}^{v}\left(\kappa_{1}, \kappa_{2}\right)=\frac{1}{\kappa_{2}} C_{r+1} Z_{2 r+2}^{s}\left(1 \mid 1 ; \kappa_{1}, \kappa_{2}\right) .
$$

Therefore, we now have expressions (4.62) and (4.63) for the partition function of vesicles with free ends, of even and odd lengths, in terms of single-walk partition functions and fixedend vesicle partition functions where the beginning and end points are at $x_{i}=x_{f}=1$.

4.1.5. Solving for the generating function of damp vesicles. The generating function

$$
G\left(z ; \kappa_{1}, \kappa_{2}\right)=\sum_{r=0}^{\infty} \dot{Z}_{2 r}^{v}\left(\kappa_{1}, \kappa_{2}\right) z^{2 r}+\sum_{r=0}^{\infty} \dot{Z}_{2 r+1}^{v}\left(\kappa_{1}, \kappa_{2}\right) z^{2 r+1}
$$

using (4.62) and (4.63), is given by

$G\left(z ; \kappa_{1}, \kappa_{2}\right)=\frac{1}{\kappa_{2}} \sum_{r=0}^{\infty} C_{r+1}\left[Z_{2 r}^{s} z^{2 r}+Z_{2 r+2}^{s} z^{2 r+1}\right]+\frac{\kappa_{2}-1}{\kappa_{2}} \sum_{r=0}^{\infty} Z_{2 r}^{\mathcal{T}} z^{2 r}$,

where $Z_{2 r}^{s} \equiv Z_{2 r}^{s}\left(1 \mid 1 ; \kappa_{1}, \kappa_{2}\right)$ as given by equation (4.40), and $Z_{2 r}^{\mathcal{T}} \equiv Z_{2 r}^{\mathcal{T}}(1 \mid 1)$.

To find our generating function $G\left(z ; \kappa_{1}, \kappa_{2}\right)$ we must find the generating function $\sum_{r=0}^{\infty} Z_{2 r}^{\mathcal{T}} z^{2 r}$ which we shall do using the recurrence (4.52).

Let us define

$$
H(z)=\sum_{r=0}^{\infty} Z_{2 r}^{\mathcal{T}} z^{2 r}
$$

and

$$
g_{r}=\left|\begin{array}{cc}
C_{r} & Z_{2 r}^{s} \\
C_{r+1} & Z_{2 r+2}^{s}
\end{array}\right|
$$


Multiplying (4.52) by $z^{2 r}$ and summing over $r$ gives us

$$
\begin{aligned}
& H(z)=\frac{\omega_{c}-\omega_{d}}{\kappa_{2}(c-d)} \sum_{r=0}^{\infty} g_{r+1} z^{2 r}+\omega_{c} \omega_{d} \sum_{r=0}^{\infty} Z_{2 r+2}^{\mathcal{T}} z^{2 r} \\
& H(z)\left(1-\frac{\omega_{c} \omega_{d}}{z^{2}}\right)=\frac{\omega_{c}-\omega_{d}}{\kappa_{2}(c-d)} \sum_{r=0}^{\infty} g_{r+1} z^{2 r}-\frac{\omega_{c} \omega_{d}}{z^{2}} Z_{0}^{\mathcal{T}} \\
& H(z)=\frac{1}{\kappa_{1}^{2}\left(z^{2}-\omega_{c} \omega_{d}\right)} \sum_{r=1}^{\infty} g_{r} z^{2 r}-\frac{\kappa_{2} \omega_{c} \omega_{d}}{z^{2}-\omega_{c} \omega_{d}} .
\end{aligned}
$$

Hence,

$$
\begin{aligned}
G_{\mathrm{dv}}\left(z ; \kappa_{1}, \kappa_{2}\right) & =\frac{1}{\kappa_{2}} \sum_{r=0}^{\infty} C_{r+1}\left[Z_{2 r}^{s} z^{2 r+2}+Z_{2 r+2}^{s} z^{2 r+3}\right] \\
& +\frac{\kappa_{2}-1}{\kappa_{2} \kappa_{1}^{2}\left(z^{2}-\omega_{c} \omega_{d}\right)} \sum_{r=1}^{\infty}\left[C_{r} Z_{2 r+2}^{s}-C_{r+1} Z_{2 r}^{s}\right] z^{2 r+2}-\frac{\left(\kappa_{2}-1\right) \omega_{c} \omega_{d} z^{2}}{z^{2}-\omega_{c} \omega_{d}} .
\end{aligned}
$$

This represents the solution for the generating function of staircase polygons above a damp wall with one end fixed and the other end free.

\subsection{Percolation probability for the damp wall}

Note that if $\kappa_{1}, \kappa_{2}$ are set to the values which relate to the percolation problem:,

$$
z=p q, \quad \kappa_{1}=\frac{p_{w}}{p q}, \quad \kappa_{2}=\frac{q_{w}}{q},
$$

then the values of the other variables are

$$
c=\frac{p}{q}, \quad d=\frac{p_{w}-p}{p}
$$

and

$$
\omega_{c}=p q, \quad \omega_{d}=\frac{p}{p_{w}^{2}}\left(p_{w}-p\right) .
$$

Note in particular that $z=\omega_{c}$.

Since $z=\omega_{c}$ we have

$$
\begin{aligned}
H\left(\omega_{c}\right) & =\frac{1}{\kappa_{2} \omega_{c}(c-d)}\left(\sum_{r=0}^{\infty} g_{r} \omega_{c}^{2 r}-g_{0}\right)-\frac{\kappa_{2} \omega_{d}}{\omega_{c}-\omega_{d}} \\
& =\frac{1}{\kappa_{2} \omega_{c}(c-d)} \sum_{r=0}^{\infty} g_{r} \omega_{c}^{2 r}-\frac{\kappa_{1}+\kappa_{2}-1}{\omega_{c}(c-d)}-\frac{\kappa_{2} \omega_{d}}{\omega_{c}-\omega_{d}} \\
& =\frac{1}{\kappa_{2} \omega_{c}(c-d)} \sum_{r=0}^{\infty} g_{r} \omega_{c}^{2 r}-\frac{\kappa_{1}}{\omega_{c}(c-d)} .
\end{aligned}
$$

So, recalling the definition of $H(z)$ in (4.66), and substituting (4.67) into (4.73), we have

$$
\sum_{r=0}^{\infty} Z_{2 r}^{\mathcal{T}} \omega_{c}^{2 r}=\frac{1}{\kappa_{2} \omega_{c}(c-d)} \sum_{r=0}^{\infty}\left(C_{r} Z_{2 r+2}^{s}-C_{r+1} Z_{2 r}^{s}\right) \omega_{c}^{2 r}-\frac{\kappa_{1}}{\omega_{c}(c-d)}
$$




$$
\begin{aligned}
G\left(\omega_{c}\left(p, p_{w}\right) ;\right. & \left.\kappa_{1}\left(p, p_{w}\right), \kappa_{2}\left(p, p_{w}\right)\right)=\frac{1}{1-c d} \sum_{r=0}^{\infty} C_{r+1} Z_{2 r}^{s} \omega_{c}^{2 r} \\
& -\frac{c d}{\kappa_{2} \omega_{c}(1-c d)(c-d)} \sum_{r=0}^{\infty}\left(C_{r} Z_{2 r+2}^{s}-C_{r+1} Z_{2 r}^{s}\right) \omega_{c}^{2 r} \\
& +\frac{c d \kappa_{1}}{\omega_{c}(c-d)(1-c d)}+\frac{1}{1-c d} \sum_{r=0}^{\infty} C_{r+1} Z_{2 r+2}^{s} \omega_{c}^{2 r+1} .
\end{aligned}
$$

Noting the form of $Z_{2 r}^{s}(1 \mid 1)$ from (4.40) and (4.42), we are able to rewrite $G\left(\omega_{c}, \kappa_{1}, \kappa_{2}\right)$ as

$$
G\left(\omega_{c} ; \kappa_{1}, \kappa_{2}\right)=\theta(c-1) \mathbf{C}-\theta(d-1) \mathbf{D}+\boldsymbol{\Omega}_{\mathbf{c}}-\boldsymbol{\Omega}_{\mathbf{d}}+\frac{c d \kappa_{1}}{\omega_{c}(c-d)(1-c d)} .
$$

We define (and treat) each of $\mathbf{C}, \mathbf{D}, \boldsymbol{\Omega}_{\mathbf{c}}$ and $\boldsymbol{\Omega}_{\mathbf{d}}$ below separately, making use, where possible, of the result,

$$
\begin{aligned}
& \sum_{s=1}^{\infty} C_{s} z^{s}=\frac{1-2 z-\sqrt{1-4 z}}{2 z} \\
& \mathbf{C}=\frac{c^{2}-1}{c(c-d)}\left(\sum_{r=0}^{\infty} C_{r+1} \omega_{c}^{r}+\sum_{r=0}^{\infty} C_{r+1} \omega_{c}^{r}+\frac{c d}{\kappa_{2} \omega_{c}(c-d)}\left(\sum_{r=0}^{\infty} C_{r+1} \omega_{c}^{r}-\sum_{r=0}^{\infty} C_{r} \omega_{c}^{r-1}\right)\right) .
\end{aligned}
$$

Using (4.77), (4.70)-(4.72) and setting $q=1-p$ gives

$$
\mathbf{C}=\frac{(1-2 q)\left(q^{2}-q+q_{w}+q q_{w}-4 q^{2} q_{w}+2 q^{2} q_{w}^{2}\right)}{q q_{w}(1-q)^{2}\left(1-2 q+q q_{w}\right)^{2}} .
$$

We do not need to consider $\mathbf{D}$, as $\theta(d-1)=0$ in the region $p>\frac{1}{2}$

$$
\begin{aligned}
\boldsymbol{\Omega}_{\mathbf{c}}=\frac{1}{c-d}( & \left.\sum_{r=0}^{\infty} C_{r+1} \omega_{c}^{r} \sum_{s=r+1}^{\infty} C_{s} \omega_{c}^{s}+\sum_{r=0}^{\infty} C_{r+1} \omega_{c}^{r} \sum_{s=r+2}^{\infty} C_{s} \omega_{c}^{s}\right) \\
& +\frac{c d}{\kappa_{2} \omega_{c}(c-d)^{2}}\left(\sum_{r=0}^{\infty} C_{r+1} \omega_{c}^{r} \sum_{s=r+1}^{\infty} C_{s} \omega_{c}^{s}-\sum_{r=0}^{\infty} C_{r} \omega_{c}^{r-1} \sum_{s=r+2}^{\infty} C_{s} \omega_{c}^{s}\right) .
\end{aligned}
$$

Making use of (4.77), we can rewrite $\Omega_{\mathbf{c}}$ as

$$
\Omega_{\mathbf{c}}=J_{1}+J_{2},
$$

where

$$
J_{1}=\frac{\left(1-2 \omega_{c}-\sqrt{1-4 \omega_{c}}\right)^{2}}{4 \omega_{c}^{3}(c-d)}-\frac{c d\left(1-2 \omega_{c}-\sqrt{1-4 \omega_{c}}\right)}{2 \omega_{c}^{3} \kappa_{2}(c-d)^{2}}
$$

and $J_{2}$ will be considered later.

Using (4.70)-(4.72) and setting $q=1-p$ gives

$$
\begin{gathered}
J_{1}=\frac{q^{2}}{(1-q)^{2}\left(1-2 q+q q_{w}\right)}-\frac{q\left(q-q_{w}\right)}{q_{w}(1-q)\left(1-2 q+q q_{w}\right)^{2}} \\
\boldsymbol{\Omega}_{\mathbf{d}}=\frac{1}{c-d}\left(\sum_{r=0}^{\infty} C_{r+1}\left(\frac{\omega_{c}^{2}}{\omega_{d}}\right)^{r} \sum_{s=r+1}^{\infty} C_{s} \omega_{d}^{s}+\frac{\omega_{c}}{\omega_{d}} \sum_{r=0}^{\infty} C_{r+1}\left(\frac{\omega_{c}^{2}}{\omega_{d}}\right)^{r} \sum_{s=r+2}^{\infty} C_{s} \omega_{d}^{s}\right) \\
+\frac{c d}{\kappa_{2} \omega_{c}(c-d)^{2}}\left(\sum_{r=0}^{\infty} C_{r+1}\left(\frac{\omega_{c}^{2}}{\omega_{d}}\right)^{r} \sum_{s=r+1}^{\infty} C_{s} \omega_{d}^{s}-\frac{1}{\omega_{d}} \sum_{r=0}^{\infty} C_{r}\left(\frac{\omega_{c}^{2}}{\omega_{d}}\right)^{r} \sum_{s=r+2}^{\infty} C_{s} \omega_{d}^{s}\right) .
\end{gathered}
$$


Similarly to (4.81), we can rewrite $\Omega_{\mathbf{d}}$ by extracting the portion of (4.84) to which we can apply (4.77), and express

$$
\Omega_{\mathbf{d}}=K_{1}+K_{2},
$$

where

$$
\begin{aligned}
K_{1} & =\frac{1}{c-d}\left(1+\frac{\omega_{c}}{\omega_{d}}\right) \sum_{r=0}^{\infty} C_{r+1}\left(\frac{\omega_{c}^{2}}{\omega_{d}}\right)^{r} \sum_{s=1}^{\infty} C_{s} \omega_{d}^{s} \\
& +\frac{c d}{\kappa_{2} \omega_{c}(c-d)^{2}}\left(\sum_{r=0}^{\infty} C_{r+1}\left(\frac{\omega_{c}^{2}}{\omega_{d}}\right)^{r} \sum_{s=1}^{\infty} C_{s} \omega_{d}^{s}-\frac{1}{\omega_{d}} \sum_{r=0}^{\infty} C_{r}\left(\frac{\omega_{c}^{2}}{\omega_{d}}\right)^{r} \sum_{s=1}^{\infty} C_{s} \omega_{d}^{s}\right)
\end{aligned}
$$

and $K_{2}$ will be considered later.

Using (4.77), (4.70)-(4.72) and setting $q=1-p$ gives, with a lot of manipulation,

$$
K_{1}=-\frac{q\left(q-q_{w}\right)\left(1-q_{w}\right)^{2}}{q_{w}(1-q)\left(1-2 q+q q_{w}\right)^{2}} .
$$

Substituting (4.79), (4.83), (4.87), into (4.76) gives

$$
\begin{aligned}
G\left(p q ; \frac{p_{w}}{p q}\right. & \left., \frac{q_{w}}{q}\right)=\theta(c-1) \mathbf{C}-\theta(d-1) \mathbf{D}+\boldsymbol{\Omega}_{\mathbf{c}}-\boldsymbol{\Omega}_{\mathbf{d}}+\frac{\left(q-q_{w}\right)\left(1-q_{w}\right)}{q q_{w}(1-q)\left(1-2 q+q q_{w}\right)} \\
& =\frac{q-2 q^{2}+q_{w}-2 q q_{w}+q^{2} q_{w}}{q(1-q)^{2}\left(1-2 q+q q_{w}\right)}+J_{2}-K_{2},
\end{aligned}
$$

where $J_{2}$ and $K_{2}$ are defined in (4.80) and (4.84) respectively so that

$$
\begin{aligned}
J_{2}-K_{2}=\frac{c d}{\kappa_{2} \omega_{c}(c-d)^{2}}\left(\sum_{r=0}^{\infty} C_{r} \omega_{c}^{r-1} \sum_{s=1}^{r+1} C_{s} \omega_{c}^{s}-\sum_{r=0}^{\infty} C_{r+1} \omega_{c}^{r} \sum_{s=1}^{r} C_{s} \omega_{c}^{s}\right) \\
+\frac{1}{c-d} \sum_{r=0}^{\infty} C_{r+1}\left(\frac{\omega_{c}^{2}}{\omega_{d}}\right)^{r}\left(\sum_{s=1}^{r} C_{s} \omega_{d}^{s}+\frac{\omega_{c}}{\omega_{d}} \sum_{s=1}^{r+1} C_{s} \omega_{d}^{s}\right) \\
+\frac{c d}{\kappa_{2} \omega_{c}(c-d)^{2}}\left(\sum_{r=0}^{\infty} C_{r+1}\left(\frac{\omega_{c}^{2}}{\omega_{d}}\right)^{r} \sum_{s=1}^{r} C_{s} \omega_{d}^{s}-\frac{1}{\omega_{d}} \sum_{r=0}^{\infty} C_{r}\left(\frac{\omega_{c}^{2}}{\omega_{d}}\right)^{r} \sum_{s=1}^{r+1} C_{s} \omega_{d}^{s}\right) .
\end{aligned}
$$

With manipulation of (4.90) it can be shown that $J_{2}-K_{2}=0$.

Hence, for $p>\frac{1}{2}$ :

$$
G\left(p q ; \frac{p_{w}}{p q}, \frac{q_{w}}{q}\right)=\frac{q-2 q^{2}+q_{w}-2 q q_{w}+q^{2} q_{w}}{q(1-q)^{2}\left(1-2 q+q q_{w}\right)} .
$$

Using the relationship (3.9) between the generating function and the percolation probability we finally arrive at the main result,

$$
\begin{aligned}
P\left(p, p_{w}\right) & =1-q^{2} G\left(p q ; \frac{p_{w}}{p q}, \frac{q_{w}}{q}\right) \\
& =\frac{(1-2 q)^{2}}{(1-q)^{2}\left(1-2 q+q q_{w}\right)} .
\end{aligned}
$$

We can also check with great difficulty that $P\left(p, p_{w}\right)=0$ for $p \leqslant 1 / 2$. 


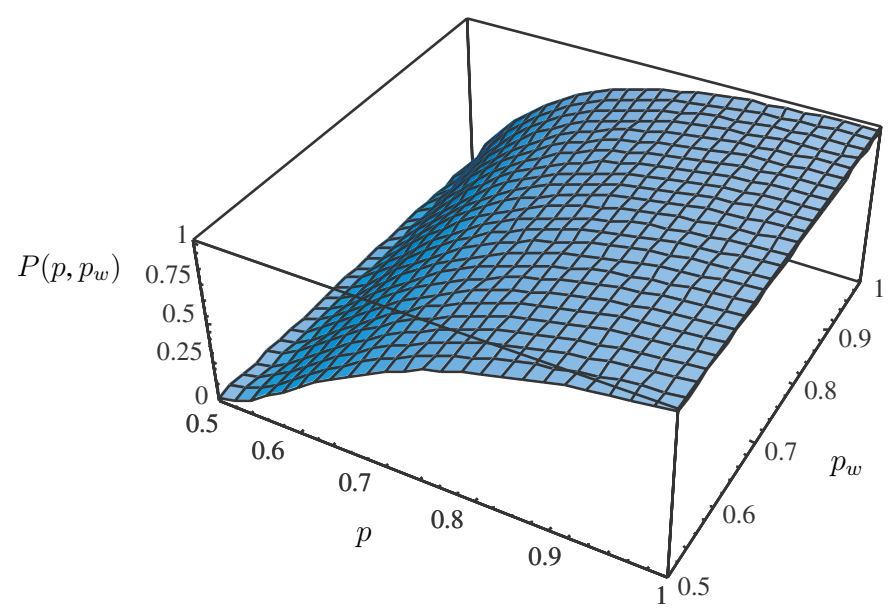

Figure 5. $P\left(p, p_{w}\right)$ plotted over $p \in\left[\frac{1}{2}, 1\right], p_{w} \in\left[\frac{1}{2}, 1\right]$.

We have hence arrived at the percolation probability for the damp case (plotted in figure 5)

$$
P\left(p, p_{w}\right)= \begin{cases}\frac{(1-2 q)^{2}}{(1-q)^{2}\left(1-2 q+q q_{w}\right)}, & p>\frac{1}{2} \\ 0 & p \leqslant 0 .\end{cases}
$$

We now check that the expression we have found for the damp percolation probability is consistent with the previously found wet and dry cases. The wet case corresponds to $p_{w}=1$, giving

$$
P(p, 1)=\frac{(1-2 q)}{(1-q)^{2}},
$$

which is the same as the result obtained for the wet case.

The dry case corresponds to $p_{w}=0$ giving

$$
P(p, 0)=\frac{(1-2 q)^{2}}{(1-q)^{3}}
$$

which is the same as the result obtained for the dry case.

We can also note from the damp case that the exponent $(\beta=2)$ is the same as in the dry case, and the transition value of $p=\frac{1}{2}$ remains unchanged by the value of $p_{w}$, which is a surface effect.

\section{Functional-equation approach}

In this section we use a functional-equation approach to compute the percolation probability. While this calculation reproduces the results from section 4 , it is a new application of the family of techniques known as the 'kernel method'. These techniques have been used to great effect in combinatorics and indeed one can see that the resulting calculations are somewhat simpler.

The main drawback of this approach is that we have not yet been able to extend this technique to other quantities of interest such as the mean-cluster length. While it is in 
principle possible to use the kernel method to obtain the generating function of the twowalker two-interaction system, we have not yet done so due to the complexity of the resulting expressions. We include both approaches here to demonstrate their strengths and weaknesses.

\subsection{Functional equation for the two-walker system}

In order to apply the functional equation method we construct the two-walker system columnby-column. In particular, each configuration in the two walker system is either of length zero, or can be constructed by appending a step to each walk of a shorter configuration. To this end we define $f\left(s, r ; z, \kappa_{1} \kappa_{2}\right)$ to be the generating function for the number of configurations of two directed walkers above a wall starting from $(0,0)$ and $(0,2)$. Note that this differs from the definition of the walks enumerated by $G$ by one pair of steps. The variable $s$ is conjugate to the height of the lower walker above the wall and $r$ is conjugate to the separation of the end points minus 2. In the text below we will often use the shorter notation $f(s, r) \equiv f\left(s, r ; z, \kappa_{1}, \kappa_{2}\right)$. We will also use the notation $\left[s^{\ell}\right]\{f(s, r)\}$ to denote the coefficient of $s^{\ell}$ in the generating function $f(s, r)$ and similarly for coefficients of $r$.

In order to compute the percolation probability we need to recover the generating function $G\left(z ; \kappa_{1}, \kappa_{2}\right)$. This is given by those two-walker configurations in which the walkers end together at any non-negative height. The connection between the two sets of walks gives $f\left(1,0 ; \kappa_{1}, \kappa_{2}\right)=1+z G\left(z ; \kappa_{1}, \kappa_{2}\right)$. The percolation probability is therefore given by

$$
P\left(p, p_{w}\right)=1-\frac{q}{p}\left(f\left(1,0 ; p q, \frac{p_{w}}{p q}, \frac{q_{w}}{q}\right)-1\right) .
$$

We now derive a functional equation for $f$. Each configuration is either a single vertexthis contributes 1 to the generating function, or is obtained by appending a single step to the end of each walk in a smaller configuration. There are four possible ways in which a single step can be appended to the end of each path-two north east steps, two south east steps and one north east step and one south east step (in two different ways). This gives $z(s+s / r+r / s+1 / s) \cdot f(s, r)$. However, in appending steps in this way, we have produced invalid configurations and we must subtract off these contributions.

- Appending a south east step to the lower walk when it finishes on the wall results in an invalid configuration (no matter which step is added to the upper walk) since the resulting walk steps below the wall. This gives $-z \frac{1+r}{s} \cdot\left[s^{0}\right]\{f(s, r)\}$.

- Appending a north east step to the lower walk and a south east step to the upper walk of a configuration whose endpoints are touching results in an invalid configuration since the walkers cross. This gives $-z \frac{s}{r} \cdot\left[r^{0}\right]\{f(s, r)\}$.

This construction results in the functional equation

$$
\begin{aligned}
f(s, r)=1+ & z\left(s+\frac{s}{r}+\frac{r}{s}+\frac{1}{s}\right) f(s, r)-\frac{z(1+r)}{s} \cdot\left[s^{0}\right]\{f(s, r)\} \\
& -\frac{z s}{r} \cdot\left[r^{0}\right]\{f(s, r)\}+\text { interaction terms. }
\end{aligned}
$$

We now take care of the interaction terms. In order to do this we subtract off the terms that have not yet been weighted by the interaction terms and add back the same contributions but with additional factors of $\kappa_{1}$ and $\kappa_{2}$ as necessary.

- When the lower walk steps south east from height 1 onto the wall the weight of the configuration should increase by a single power of $\kappa_{1}$ (no matter which step is appended to the other walk). This gives $z\left(\kappa_{1}-1\right)(1+r) \cdot\left[s^{1}\right]\{f(s, r)\}$. 
- Similarly when the lower walk steps north east from height 0 or south east from height 2 to end at height 1 , then the weight of the configuration should increase by a single power of $\kappa_{2}$. This gives $z\left(\kappa_{2}-1\right) s(1+r) \cdot\left[s^{2}\right]\{f(s, r)\}+z\left(\kappa_{2}-1\right) s\left(1+\frac{1}{r}\right) \cdot\left[s^{0}\right]\{f(s, r)\}$.

- However this last term possibly reintroduces configurations in which the walkers cross and so these must be subtracted off again to give $-z\left(\kappa_{2}-1\right) \frac{s}{r} \cdot\left[s^{0} r^{0}\right]\{f(s, r)\}$.

Putting these terms together and rewriting the coefficients in terms of derivatives gives

$$
\begin{aligned}
f(s, r)=1+ & z\left(s+\frac{s}{r}+\frac{r}{s}+\frac{1}{s}\right) f(s, r)-\frac{z s}{r} f(s, 0)-\frac{z(1+r)}{s} f(0, r) \\
& +z\left(\kappa_{1}-1\right)(1+r) \frac{\partial f}{\partial s}(0, r)+z\left(\kappa_{2}-1\right) \frac{s(1+r)}{2} \frac{\partial^{2} f}{\partial s^{2}}(0, r) \\
& +z\left(\kappa_{2}-1\right) s\left(1+\frac{1}{r}\right) f(0, r)-z\left(\kappa_{2}-1\right) \frac{s}{r} f(0,0) .
\end{aligned}
$$

We can now simplify this equation and remove the two unknowns $\frac{\partial f}{\partial s}(0, r)$ and $\frac{\partial^{2} f}{\partial s^{2}}(0, r)$ by differentiating equation (5.3) repeatedly and setting $s=0$. Differentiating once and setting $s=0$ gives

$$
z \kappa_{1}(1+r) \frac{\partial f}{\partial s}(0, r)=f(0, r)-1
$$

Differentiating twice and setting $s=0$ gives

$z \kappa_{2} r(1+r) \frac{\partial^{2} f}{\partial s^{2}}(0, r)=2\left(r \frac{\partial f}{\partial s}(0, r)-z \kappa_{2}(1+r) f(0, r)+z \kappa_{2} f(0,0)\right)$.

Substituting both these expressions back into equation (5.3) and multiplying by $s r$ gives the following expression:

$$
f(s, r) K(s, r)=A(s, r)+B(s, r) f(s, 0)+C(s, r) f(0, r),
$$

where

$$
\begin{aligned}
& K(s, r)=s r-z\left(s^{2} r+s^{2}+r^{2}+r\right), \\
& A(s, r)=\frac{s r}{\kappa_{1}}-\frac{s^{2} r\left(\kappa_{2}-1\right)}{z \kappa_{1} \kappa_{2}(1+r)}, \\
& B(s, r)=-z s^{2}, \\
& C(s, r)=-z r(1+r)+\frac{\left(\kappa_{1}-1\right) s r}{\kappa_{1}}+\frac{\left(\kappa_{2}-1\right) s^{2} r}{z \kappa_{1} \kappa_{2}(1+r)} .
\end{aligned}
$$

The polynomial $K(s, r)$ is called the kernel. Note that this new equation does not contain the term $f(0,0)$; it has been cancelled in the process of removing $\frac{\partial f}{\partial s}(0, r)$ and $\frac{\partial^{2} f}{\partial s^{2}}(0, r)$.

Rather than solving this equation directly to obtain $f(s, r)$, we will first map to the percolation problem and solve for $f(1,0)$ using the kernel method. The above functional equation contains several unknown functions; by making careful choices of $s$ and $r$ we can cancel the kernel and generate new equations that allow us to eliminate these unknown functions and so solve for $f(1,0)$.

Recall that we map to the percolation problem by setting

$$
z \mapsto p q, \quad \kappa_{1} \mapsto \frac{p_{w}}{p q_{w}}, \quad \kappa_{2} \mapsto \frac{q_{w}}{q},
$$

where $q=1-p$ and $q_{w}=1-p_{w}$. Setting $p_{w}=p$ returns us to the dry-wall case $\left(\kappa_{1}=1 / q, \kappa_{2}=1\right)$.

We first solve the equation in the case of small $p$ and then solve in the case of small $q$. We do not need the full $f(s, r)$ generating function; we only require $f(1,0)$ since it counts configurations with adjacent endpoints but lying at any distance above the wall. 


\subsection{Small $p$}

Consider the functional equation after mapping to the percolation problem and with all $q$ and $q_{w}$ terms rewritten in terms of $p$ and $p_{w}$. This gives

$$
\begin{aligned}
& K(s, r)=s r-p(1-p)\left(s^{2} r+s^{2}+r^{2}+r\right) \\
& A(s, r)=\frac{\operatorname{srp}\left(p_{w}-1\right)}{p_{w}}+\frac{s^{2} r\left(p-p_{w}\right)}{(1+r)(p-1) p_{w}} \\
& B(s, r)=-p(1-p) s^{2} \\
& C(s, r)=-p(1-p) r(1+r)+\frac{s r\left(p_{w}-p+p p_{w}\right)}{p_{w}}-\frac{s^{2} r\left(p-p_{w}\right)}{(1+r)(1-p) p_{w}} .
\end{aligned}
$$

If we try to set $s=1, r=0$ in the functional equation then we obtain the tautology $f(1,0)=f(1,0)$.

Instead we set $s=1$ and then look for values of $r$ that set $K(s, r)=0$; this is the idea of the kernel method. Doing so gives two solutions $r=\frac{p}{1-p}, \frac{1-p}{p}$. Since we wish to obtain a solution that is valid for small $p$, we cannot substitute the second of these (it will result in a power series that is singular as $p \rightarrow 0$ ). When we substitute $s=1, r=\frac{p}{1-p}, K$ is zero and by happy coincidence, $C$ is also zero; we are left with

$$
0=p-p(1-p) f(1,0) .
$$

So $f(1,0)=\frac{1}{1-p}$ and thus the percolation probability is given by

$$
P(p)=1-\frac{1-p}{p}(f(1,0)-1)=0
$$

as is expected for small $p$.

\subsection{Small $q$}

Now consider equation (5.6) mapped to the percolation problem and with everything written in terms of $q, q_{w}$ :

$$
\begin{aligned}
& K(s, r)=s r-q(1-q)\left(s^{2} r+s^{2}+r^{2}+r\right) \\
& A(s, r)=\frac{s r(1-q) q_{w}}{\left(1-q_{w}\right)}+\frac{s^{2} r\left(q-q_{w}\right)}{(1+r) q\left(1-q_{w}\right)} \\
& B(s, r)=-q(1-q) s^{2} \\
& C(s, r)=-q(1-q) r(1+r)+\frac{s r\left(1-2 q_{w}+q q_{w}\right)}{1-q_{w}}-\frac{s^{2} r\left(q-q_{w}\right)}{(1+r) q\left(1-q_{w}\right)(1+r)} .
\end{aligned}
$$

The kernel method does not work as cleanly in this case and we must make several different substitutions for $s, r$ in order to obtain $f(1,0)$.

- As above, we start by setting $s=1$ and $r=\frac{q}{1-q}$ which cancels $K$,

$$
\frac{(1-2 q) q_{w}}{\left(1-q_{w}\right)} f\left(0, \frac{q}{1-q}\right)+\frac{q+q q_{w}-q_{w}}{1-q_{w}}-q(1-q) f(1,0) .
$$

We will return to this equation after we have found $f\left(0, \frac{q}{1-q}\right)$. 
- Since we have $f(1,0)$ in terms of $f\left(0, \frac{q}{1-q}\right)$, we next set $r=\frac{q}{1-q}$. We see that there are two values of $s$ that cancel $K$, namely $s=1, \frac{q}{1-q}$. Setting $s=r=\frac{q}{1-q}$ cancels both $K$ and $C$ :

$$
f\left(\frac{q}{1-q}, 0\right)=\frac{1}{1-q} .
$$

- Since we have $f\left(\frac{q}{1-q}, 0\right)$ in closed form, set $s=\frac{q}{1-q}$ and leave $r=r$. This results in an ugly expression involving $f\left(\frac{q}{1-q}, r\right), f\left(\frac{q}{1-q}, 0\right)$ and $f(0, r)$.

Substitute $f\left(\frac{q}{1-q}, 0\right)=\frac{1}{1-q}$ into this expression and then remove the resulting common factor of $(q-r(1-q))$. This leaves an equation in $f\left(\frac{q}{1-q}, r\right)$ and $f(0, r)$. The coefficient of the first of these terms can be cancelled by setting $r=\frac{q}{1-q}$. This leaves

$$
f\left(0, \frac{q}{1-q}\right)=\frac{1-q}{1-2 q+q_{w}}
$$

which is precisely what we require to find $f(1,0)$.

- Substitute this into equation (5.13) to obtain

$$
f(1,0)=\frac{1-2 q+q_{w}-q q_{w}}{(1-q)\left(1-2 q+q q_{w}\right)} .
$$

The percolation probability is therefore

$$
P\left(q, q_{w}\right)=1-\frac{q}{1-q}(f(1,0)-1)=\frac{(1-2 q)^{2}}{(1-q)^{2}\left(1-2 q+q q_{w}\right)},
$$

which is precisely the result obtained in equation (4.93).

\section{Discussion}

The next natural step in this work will be to find the mean length and mean number of contacts for finite clusters near a damp wall. It is anticipated that this will be able to be achieved using similar methods to those used to find these properties in the dry case [5]. This will involve finding differential equations satisfied by the cluster properties, and using Zeilberger's algorithm to allow us to compute derivatives of the vesicle partition function, and hence calculate the mean length of finite clusters and mean number of surface contacts.

Following this work, there are many other directions which could be pursued in this area of research. For example, we have so far only considered the damp case for a seed width $(\mathrm{m})$ of one, so it might be of interest to investigate the percolation probability for general $\mathrm{m}$. Another variation on this problem would be to investigate the effect of having a bias: that is, assign a probability $p_{u}$ for 'up' steps, and a probability $p_{d}$ for 'down' steps, rather than the same probability $p$ for both. It would also be possible to consider the effect of more than 1 wall, or perhaps a wall that was not perpendicular to the direction of movement of the cluster.

\section{Acknowledgments}

Financial support from the Australian Research Council via its support for the Centre of Excellence for Mathematics and Statistics of Complex Systems is gratefully acknowledged by the authors. The authors would also like to thank NSERC of Canada for financial support. 


\section{References}

[1] Ball Z, Phillips H M, Callahan D L and Sauerbrey R 1994 Percolative metal-insulator transition in excimer-laser irradiated polyimide Phys. Rev. Lett. 73 2099-102

[2] Bidaux R and Privman V 1991 Surface-to-bulk crossover in directed compact percolation J. Phys. A: Math. Gen. 24 L839-43

[3] Bousquet-Mèlou M, Guttmann A J, Orrick W P and Rechnitzer A 1999 Inversion relations, reciprocity and polyominoes Ann. Combinat. $3223-49$

[4] Brak R and Essam J W 2004 Asymmetric exclusion model and weighted lattice paths J. Phys. A: Math. Gen. 37 4183-217

[5] Brak R and Essam J W 1999 Directed compact percolation near a wall: III. Exact results for the mean length and number of contacts J. Phys. A: Math. Gen. 32 353-67

[6] Brak R and Guttmann A J 1990 Exact solution of the staircase and row-convex polygon perimeter and area generating function J. Phys. A: Math. Gen. 23 4581-8

[7] Cardy J L and Grassberger P 1985 Epidemic models and percolation J. Phys. A: Math. Gen. 18 L267

[8] Domany E and Kinzel W 1984 Equivalence of cellular automata to Ising models and directed percolation Phys. Rev. Lett. $53311-4$

[9] Durrett R 1984 Oriented percolation in two dimensions Ann. Prob. 12 999-1040

[10] Essam J W 1989 Directed compact percolation: cluster size and hyperscaling J. Phys. A: Math. Gen. 22 4927-37

[11] Essam J W and Guttmann A J 1995 Directed compact percolation near a wall: II. Cluster length and size J. Phys. A: Math. Gen. 28 3591-8

[12] Essam J W, Guttmann A J, Jensen I and TanlaKishani D 1996 Directed percolation near a wall J. Phys. A: Math. Gen. 29 1619-28

[13] Essam J W and TanlaKishani D 1990 Disorder in Physical Systems ed G R Grimmett and D J A Welsh (Oxford: Oxford University Press)

[14] Essam J W and TanlaKishani D 1994 Directed compact percolation near a wall: I. Biased growth J. Phys. A: Math. Gen. 27 3743-50

[15] Gessel I M and Viennot G 1985 Binomial determinants, paths, and hook length formulae Adv. Math. 58300321

[16] Gessel I M and Viennot X 1989 Determinants, paths, and plane partitions (preprint)

[17] Hinrichsen H, Jiménez-Dalmaroni A, Rozov Y and Domany E 1999 Flowing sand: a physical realization of directed percolation Phys. Rev. Lett. 83 4999-5002

[18] Lin J C 1992 Exact results for directed compact percolation near a nonconducting wall Phys. Rev. A 45 R3394-7

[19] Stauffer D and Aharony A 1991 Introduction to Percolation Theory 2nd edn (London: Taylor and Francis)

[20] Zeilberger D 1990 A fast algorithm for proving terminating hypergeometric identities Discrete Math. 80 207-11

[21] Zeilberger D 1991 The method of creative telescoping J. Symb. Comput. 11 195-204 